\title{
Cyclic homology, tight crossed products, and small stabilizations
}

\author{
Guillermo Cortiñas*
}

\begin{abstract}
In [1] (arXiv:1212.5901) we associated an algebra $\Gamma^{\infty}(\mathfrak{A})$ to every bornological algebra $\mathfrak{A}$ and an ideal $I_{S(\mathfrak{A})} \triangleleft \Gamma^{\infty}(\mathfrak{A})$ to every symmetric ideal $S \triangleleft \ell^{\infty}$. We showed that $I_{S(\mathfrak{A})}$ has $K$-theoretical properties which are similar to those of the usual stabilization with respect to the ideal $J_{S} \triangleleft \mathcal{B}$ of the algebra $\mathcal{B}$ of bounded operators in Hilbert space which corresponds to $S$ under Calkin's correspondence. In the current article we compute the relative cyclic homology $H C_{*}\left(\Gamma^{\infty}(\mathfrak{A}): I_{S(\mathfrak{A})}\right)$. Using these calculations, and the results of loc. cit., we prove that if $\mathfrak{A}$ is a $C^{*}$-algebra and $c_{0}$ the symmetric ideal of sequences vanishing at infinity, then $K_{*}\left(I_{c_{0}(\mathfrak{A})}\right)$ is homotopy invariant, and that if $* \geq 0$, it contains $K_{*}^{\text {top }}(\mathfrak{A})$ as a direct summand. This is a weak analogue of the Suslin-Wodzicki theorem ([20]) that says that for the ideal $\mathcal{K}=J_{c_{0}}$ of compact operators and the $C^{*}$-algebra tensor product $\mathfrak{A} \widetilde{\otimes} \mathcal{K}$, we have $K_{*}(\mathfrak{A} \widetilde{\otimes} \mathcal{K})=K_{*}^{\text {top }}(\mathfrak{A})$. Similarly, we prove that if $\mathfrak{A}$ is a unital Banach algebra and $\ell^{\infty-}=\bigcup_{q<\infty} \ell^{q}$, then $K_{*}\left(I_{\ell} \infty-(\mathfrak{A})\right)$ is invariant under Hölder continuous homotopies, and that for $* \geq 0$ it contains $K_{*}^{\text {top }}(\mathfrak{A})$ as a direct summand. These $K$-theoretic results are obtained from cyclic homology computations. We also compute the relative cyclic homology groups $H C_{*}\left(\Gamma^{\infty}(\mathfrak{A}): I_{S(\mathfrak{A})}\right)$ in terms of $H C_{*}\left(\ell^{\infty}(\mathfrak{A}): S(\mathfrak{A})\right)$ for general $\mathfrak{A}$ and $S$. For $\mathfrak{A}=\mathbb{C}$ and general $S$, we further compute the latter groups in terms of algebraic differential forms. We prove that the map $H C_{n}\left(\Gamma^{\infty}(\mathbb{C}): I_{S(\mathbb{C})}\right) \rightarrow H C_{n}\left(\mathcal{B}: J_{S}\right)$ is an isomorphism in many cases.
\end{abstract}

Mathematics Subject Classification (2010). 19D55; 47L20.

Keywords. operator ideal, Calkin's theorem, crossed product, Karoubi's cone, cyclic homology.

\section{Introduction}

Let $\ell^{2}=\ell^{2}(\mathbb{N})$ be the Hilbert space of square-summable sequences of complex numbers and $\mathcal{B}=\mathcal{B}\left(\ell^{2}\right)$ the algebra of bounded operators. Calkin's theorem in [3, Theorem 1.6], as restated by Garling in [15, Theorem 1], establishes an isomorphism

$$
S \mapsto J_{S}
$$

\footnotetext{
* Research supported by CONICET and by grants UBACyT W386, PIP 112-200801-00900, MTM2007-64704 (FEDER funds) and by MathAmSud network U11MATH-05. The latter network was partially funded by ANII, Uruguay, and by MINCyT, Argentina.
} 
between the lattice of proper symmetric ideals of the algebra $\ell^{\infty}$ of bounded sequences and that of proper two-sided ideals of the algebra $\mathcal{B}=\mathcal{B}\left(\ell^{2}\right)$ of bounded operators. In [1] we introduced a subalgebra $\Gamma^{\infty} \subset \mathcal{B}$ and showed that the above lattices are also isomorphic to the lattice of proper two-sided ideals of $\Gamma^{\infty}$, via the correspondence

$$
S \mapsto I_{S}=J_{S} \cap \Gamma^{\infty} .
$$

More generally, we associated to each bornological algebra $\mathfrak{A}$, an algebra $\Gamma^{\infty}(\mathfrak{A})$ which contains an ideal $I_{S(\mathfrak{A})}$ for each symmetric ideal $S \triangleleft \ell^{\infty}$. We showed that the algebra $I_{S(\mathfrak{A})}$ has $K$-theoretical properties which are analogous to those of the usual stabilization with respect to $J_{S}$, at least when $S$ is one of the following:

$$
S \in\left\{c_{0}, \ell^{p-}, \ell^{q}, \ell^{q+} \quad(p \leq \infty, q<\infty)\right\} .
$$

Here $c_{0}$ is the ideal of sequences vanishing at infinity, $\ell^{q}$ consists of the $q$-summable sequences, and

$$
\ell^{p-}=\bigcup_{r<p} \ell^{r}, \quad \ell^{q+}=\bigcap_{s>q} \ell^{s} .
$$

We proved that for $S$ as in (1.1), there is a long exact sequence:

$$
\begin{aligned}
K H_{n+1}\left(I_{S(\mathfrak{A})}\right) & \longrightarrow H C_{n-1}\left(\Gamma^{\infty}(\mathfrak{A}): I_{S(\mathfrak{A})}\right) \\
& \downarrow \\
K H_{n}\left(I_{S(\mathfrak{A})}\right) & \longleftarrow
\end{aligned}
$$

If furthermore, $S \neq c_{0}$, then $K H_{*}\left(I_{S(\mathfrak{A})}\right)=K H_{*}\left(I_{\ell^{1}(\mathfrak{R})}\right)$. We proved that the functor $K H_{*}\left(I_{c_{0}(\mathfrak{A})}\right)$ is invariant under arbitrary continuous homotopies of bornological algebras, and that $K H_{*}\left(I_{\ell^{1}(\mathfrak{L})}\right)$ is invariant under Hölder continuous homotopies. We also showed that if $* \geq 0$ and either $\mathfrak{A}$ is a $C^{*}$-algebra and $S=c_{0}$ or $\mathfrak{A}$ is a local Banach algebra and $S=\ell^{1}$, then $K H_{*}\left(I_{S(\mathfrak{R})}\right)$ contains $K_{*}^{\text {top }}(\mathfrak{A})$ as a direct summand. In the current article we study the groups $H C_{*}\left(\Gamma^{\infty}(\mathfrak{A}): I_{S(\mathfrak{A})}\right)$ for general $S$ and $\mathfrak{A}$. We show for example that if $\mathfrak{A}$ is a $C^{*}$-algebra then $I_{c_{0}(\mathfrak{A})}$ is $H$-unital and

$$
H C_{*}\left(\Gamma^{\infty}(\mathfrak{A}): I_{c_{0}(\mathfrak{A})}\right)=0 .
$$

It follows from this, excision, and the exact sequence (1.2), that the comparison map

$$
K_{*}\left(I_{c_{0}(\mathfrak{A})}\right) \rightarrow K H_{*}\left(I_{c_{0}(\mathfrak{A})}\right)
$$

is an isomorphism. In particular, if $\mathfrak{A}$ is a $C^{*}$-algebra, then $K_{*}\left(I_{c_{0}(\mathfrak{A})}\right)$ is homotopy invariant, and if $* \geq 0$, it contains $K_{*}^{\text {top }}(\mathfrak{A})$ as a direct summand. This again shows that $I_{c_{0}(-)}$ has properties analogous to those of $J_{c_{0}}=\mathcal{K}$, the ideal of 
compact operators. Indeed, the result above is a weak analogue of the SuslinWodzicki theorem (Karoubi's conjecture) which says that if $\mathfrak{A}$ is a $C^{*}$-algebra then $K_{*}(\mathfrak{A} \widetilde{\otimes} \mathcal{K})=K_{*}^{\text {top }}(\mathfrak{A})$. We also show that if $\mathfrak{A}$ is a unital Banach algebra then $I_{\ell \infty-(\mathfrak{A})}$ is $H$-unital and

$$
H C_{*}\left(\Gamma^{\infty}(\mathfrak{A}): I_{\ell}{ }^{\infty-(\mathfrak{A})}\right)=0 .
$$

Thus the comparison map

$$
K_{*}\left(I_{\ell \infty-(\mathfrak{A})}\right) \rightarrow K H_{*}\left(I_{\ell \infty-(\mathfrak{A})}\right)
$$

is an isomorphism. Again this is analogous to a similar property of stabilization with respect to $J_{\ell \infty-}=\bigcup_{p} \mathcal{L}^{p}$, the union of all Schatten ideals (see [24, pp. 490], [9, Theorem 8.2.5]). In [24], M. Wodzicki studied the relative cyclic homology groups $H C_{n}\left(\mathcal{B}: J_{S}\right)$. For $S$ as in (1.1), the following integer was computed by Wodzicki in [24, Corollary to Theorem 8]

$$
m=m_{S}=\min \left\{n: H C_{n}\left(\mathcal{B}: J_{S}\right) \neq 0\right\} .
$$

We prove in Proposition 7.3 that

$$
m=\min \left\{n: H C_{n}\left(\Gamma^{\infty}: I_{S}\right) \neq 0\right\},
$$

and that the natural map is an isomorphism for $n=m$ :

$$
H C_{m}\left(\Gamma^{\infty}: I_{S}\right) \stackrel{\cong}{\longrightarrow} H C_{m}\left(\mathcal{B}: J_{S}\right) .
$$

The techniques used in this article to establish the results above about $H C_{*}\left(\Gamma^{\infty}(\mathfrak{A})\right.$ : $\left.I_{S(\mathfrak{A})}\right)$ are similar to those used in [24] to study the relative cyclic homology of stabilizations by $J_{S}$. We also obtain more results about the groups $H C_{*}\left(\Gamma^{\infty}(\mathfrak{A})\right.$ : $\left.I_{S(\mathfrak{A})}\right)$ using a different technique, which involves a description of $\Gamma^{\infty}$ and $I_{S}$ as crossed products, established in [1, Proposition 6.12]. The inverse monoid Emb of all partially defined injections

$$
\mathbb{N} \supset \operatorname{dom} f \stackrel{f}{\longrightarrow} \mathbb{N} .
$$

acts on $\ell^{\infty}(\mathfrak{A})$ by

$$
f_{*}(\alpha)_{n}=\left\{\begin{array}{cc}
\alpha_{m} & \text { if } f(m)=n \\
0 & \text { else. }
\end{array}\right.
$$

By definition, an ideal $S \triangleleft \ell^{\infty}$ is symmetric if the action above maps $S$ to itself. Observe that if $A, B \subset \mathbb{N}$ are disjoint then the inclusions $p_{A}: A \rightarrow \mathbb{N}$ and $p_{B}$ : $B \rightarrow \mathbb{N}$ satisfy

$$
\left(p_{A \cup B}\right)_{*}=\left(p_{A}\right)_{*}+\left(p_{B}\right)_{*}
$$


In other words, the action above is tight in the sense of Exel [14]. Thus $\ell^{\infty}(\mathfrak{A})$ is a module over the ring

$$
\Gamma=\mathbb{Z}[\mathrm{Emb}] /\left\langle p_{A}+p_{B}-p_{A \cup B}: A \cap B=\emptyset\right\rangle
$$

Let $\mathcal{P} \subset \Gamma$ be the subring generated by all the $p_{A}$ with $A \subset \mathbb{N}$. Note that $\mathcal{P}$ is isomorphic to the subring of $\ell^{\infty}(\mathfrak{A})$ consisting of those sequences $\alpha: \mathbb{N} \rightarrow \mathbb{Z}$ which take finitely many distinct values. In particular (1.7) makes $\mathcal{P}$ into a $\Gamma$-module. Moreover $\ell^{\infty}(\mathfrak{A})$ is a $\mathcal{P}$-algebra, and the map

$$
H C\left(\ell^{\infty}(\mathfrak{A}): S(\mathfrak{A})\right) \rightarrow H C\left(\left(\ell^{\infty}(\mathfrak{A}) / \mathcal{P}: S(\mathfrak{A})\right) / \mathcal{P}\right)
$$

is a quasi-isomorphism (see Example 6.6 and (6.6.3)). Furthermore the action of Emb on $\ell^{\infty}(\mathfrak{A})$ extends to a tight action on $H C\left(\ell^{\infty}(\mathfrak{A}): I_{S(\mathfrak{A})}\right)$, and we show that

$$
H C_{*}\left(\Gamma^{\infty}(\mathfrak{A}): I_{S(\mathfrak{A})}\right)=\mathbb{H}_{*}\left(\Gamma / \mathcal{P}: H C\left(\left(\ell^{\infty}(\mathfrak{A}): S(\mathfrak{A})\right) / \mathcal{P}\right)\right) .
$$

Here the hyperhomology groups $\mathbb{H}_{*}(\Gamma / \mathcal{P},-)$ are the hyperderived functors of the functor

$$
\Gamma-\operatorname{Mod} \rightarrow \mathfrak{A b}, \quad M \mapsto H_{0}\left(\Gamma^{\infty} / \mathcal{P}, M\right):=M \otimes_{\Gamma} \mathcal{P} .
$$

We show in Proposition 6.3 that

$$
\begin{aligned}
& H_{0}(\Gamma / \mathcal{P}, M)=M_{\mathcal{E}} \\
& \quad=M / \operatorname{span}\left\{m-f_{*}(m): m \in M, f \in \text { Emb such that } \operatorname{dom} f=\mathbb{N}\right\} .
\end{aligned}
$$

It follows from (1.8) and (1.9) that there is a first quadrant spectral sequence

$$
E_{p, q}^{2}=H_{p}\left(\Gamma / \mathcal{P}, H C_{q}\left(\ell^{\infty}(\mathfrak{A}): S(\mathfrak{A})\right)\right) \Rightarrow H C_{p+q}\left(\Gamma^{\infty}(\mathfrak{A}): I_{S(\mathfrak{A})}\right) .
$$

In particular

$$
H C_{0}\left(\left(\Gamma^{\infty}(\mathfrak{A}): I_{S(\mathfrak{A})}\right)=H_{0}\left(\Gamma / \mathcal{P}: \ell^{\infty}(\mathfrak{A}) /\left[\ell^{\infty}(\mathfrak{A}): S(\mathfrak{A})\right]\right) .\right.
$$

Specializing to $\mathfrak{A}=\mathbb{C}$ and using (1.10) and [13, Theorem 5.12] we obtain

$$
H C_{0}\left(\Gamma^{\infty}: I_{S}\right)=S_{\mathcal{E}}=H C_{0}\left(\mathcal{B}: J_{S}\right)
$$

for every symmetric ideal $S \triangleleft \ell^{\infty}$. Another application of (1.9) is that for $\mathfrak{A}$ commutative the groups $H C_{*}\left(\Gamma^{\infty}(\mathfrak{A}): I_{S(\mathfrak{A})}\right)$ carry a natural Hodge decomposition. Indeed, the usual Hodge decomposition of the cyclic chain complex [17] gives an Emb-equivariant direct sum decomposition

$$
H C\left(\left(\ell^{\infty}(\mathfrak{A}): S(\mathfrak{A})\right) / \mathcal{P}\right)=\bigoplus_{p \geq 0} H C^{(p)}\left(\left(\ell^{\infty}(\mathfrak{A}): S(\mathfrak{A})\right) / \mathcal{P}\right) .
$$


Thus for

$$
H C^{(p)}\left(\Gamma^{\infty}(\mathfrak{A}): I_{S(\mathfrak{A})}\right)=\mathbb{H}\left(\Gamma / \mathcal{P}, H C^{(p)}\left(\left(\ell^{\infty}(\mathfrak{A}): S(\mathfrak{A})\right) / \mathcal{P}\right)\right)
$$

we have

$$
H C_{n}\left(\Gamma^{\infty}(\mathfrak{A}): I_{S(\mathfrak{A})}\right)=\bigoplus_{p=0}^{n} H C_{n}^{(p)}\left(\Gamma^{\infty}(\mathfrak{A}): I_{S(\mathfrak{A})}\right) .
$$

In Theorem 7.7 we obtain a description of $H C_{n}^{(p)}\left(\Gamma^{\infty}: I_{S}\right)$ in terms of differential forms which we shall presently explain. Let $\Omega_{\ell \infty}$ be the de Rham complex of absolute -i.e. $\mathbb{Z}$-linear- algebraic differential forms. For $p \geq 0$ consider the subcomplex

$$
\left(\mathcal{F}_{p}(S)\right)^{q}=\left\{\begin{array}{cc}
S^{p-q+1} \Omega_{\ell \infty}^{q} & p \geq q \\
\Omega_{\ell \infty}^{q} & q>p .
\end{array}\right.
$$

We show in Theorem 7.7 that

$$
H C_{*}^{(p)}\left(\Gamma^{\infty}: I_{S}\right)=\mathbb{H}_{*+p}\left(\Gamma / \mathcal{P}, \mathcal{F}_{(p)}(S)\right) .
$$

It follows that there is a spectral sequence (Corollary 7.8)

$$
{ }_{p} E_{m, n}^{1}=H_{n}\left(\Gamma / \mathcal{P}, S^{m+1} \Omega_{\ell \infty}^{p-m}\right) \Rightarrow H C_{m+n+p}^{(p)}\left(\Gamma^{\infty}: I_{S}\right) .
$$

Using this spectral sequence, we obtain (Corollary 7.9)

$$
H C_{n}^{(n)}\left(\Gamma^{\infty}: I_{S}\right)=\left(S \Omega_{\ell \infty}^{n} / d\left(S^{2} \Omega_{\ell \infty}^{n-1}\right)\right)_{\mathcal{E}}
$$

for every symmetric ideal $S \triangleleft \ell^{\infty}$. In the particular cases (1.1) we can say more (see Proposition 7.12). We show, for example, that if $p \in \mathbb{Z}$, then

$$
H C_{n}^{(q)}\left(\Gamma^{\infty}: I_{\ell p}\right)=\left\{\begin{array}{cc}
0 & n<q+p-1 \\
\left(\ell^{1} \Omega_{\ell \infty}^{q-p} / d\left(\ell^{p / p+1} \Omega_{\ell \infty}^{q-p}\right)\right)_{\mathcal{E}} & n=q+p-1 .
\end{array}\right.
$$

In particular, by (1.5) and (1.6) we have

$$
H C_{2 p-2}\left(\mathcal{B}: \mathcal{L}^{p}\right)=H C_{2 p-2}\left(\Gamma^{\infty}: I_{\ell p}\right)=H C_{2 p-2}^{(p-1)}\left(\Gamma^{\infty}: I_{\ell}\right)=\ell_{\mathcal{E}}^{1} .
$$

The rest of this paper is organized as follows. In Section 2 we recall some material from [1], including, in particular, the crossed product decomposition $I_{S(\mathfrak{A})}=S(\mathfrak{A}) \#_{\mathcal{P}} \Gamma$ (Proposition 2.3). This crossed product is just the tensor product $S(\mathfrak{A}) \otimes_{\mathcal{P}} \Gamma$ with multiplication twisted by the action of Emb on $S(\mathfrak{A})$

$$
(a \# f)(b \# g)=a f_{*}(b) \# f g .
$$

In particular

$$
\left.\Gamma^{\infty}(\mathfrak{A})=I_{\ell \infty(\mathfrak{A})}=\ell^{\infty}(\mathfrak{A}) \#_{\mathcal{P}} \Gamma\right)
$$


In Section 3 we show that every two-sided ideal of $\Gamma^{\infty}$ is flat (Proposition 3.3). Furthermore, if $S$ is closed under taking square roots of positive elements (e.g. if $\left.S=c_{0}, \ell^{\infty-}\right)$ then $I_{S(\mathfrak{A})}$ is a flat ideal of $\Gamma^{\infty}(\mathfrak{A})$ for every unital Banach algebra $\mathfrak{A}$ (Proposition 3.5). Section 4 concerns the algebra $\mathcal{P}$. We show that $\mathcal{P}$ is a filtering colimit of separable $\mathbb{Z}$-algebras (Proposition 4.1) and that if $k$ is a field then $\mathcal{P}(k)=\mathcal{P} \otimes k$ is von Neumann regular (Corollary 4). Hence if $k$ is a field then every $\mathcal{P}(k)$-module is flat. Further, we show that for any unital ring $R$, $\Gamma(R)=\Gamma \otimes R$ is flat as a module over $\mathcal{P}(R)$ (Proposition 4.2). The next section concerns excision. We call a ring $A K$-excisive if it satisfies excision in algebraic $K$-theory. It was proved by Suslin and Wodzicki [20] that a ring having a certain triple factorization property (TFP) is $K$-excisive. We prove in Proposition 5.1 that if $\mathfrak{A}$ is a bornological algebra and $S \triangleleft \ell^{\infty}$ is a symmetric ideal such that $S(\mathfrak{A})$ has the TFP, then $I_{S(\mathfrak{A})}$ is $K$-excisive. This applies, for example, when $\mathfrak{A}$ is a $C^{*}$-algebra and $S=c_{0}$ (Example 5.2), and also when $\mathfrak{A}$ is a unital Banach algebra and $S=\ell^{\infty-}$ (Example 5.3). Section 6 is concerned with the homology of crossed products of the form $R \#_{\mathcal{P}} \Gamma$ where $R$ is unital. The identity (1.10) is proved in Proposition 6.3. The quasi-isomorphism (1.8) follows from the case $k=\mathbb{Q}$ of Example 6.6, which says that if $k$ is a field, $A$ is a unital $\mathcal{P}(k)$-algebra, and $N$ is an $A \otimes_{\mathcal{P}(k)} A^{o p}$-module, then the map of Hochschild complexes

$$
H H(A / k, N) \rightarrow H H(A / \mathcal{P}(k), N)
$$

is a quasi-isomorphism. In Proposition 6.7 we compute the Hochschild homology of a crossed product $R \#_{\mathcal{P}} \Gamma$ with coefficients in a bimodule of the form $M \#_{\mathcal{P}} \Gamma$. We show that there is a quasi-isomorphism

$$
\mathbb{H}(\Gamma / \mathcal{P}, H H(R / \mathcal{P}(k), M)) \stackrel{\sim}{\longrightarrow} H H\left(R \#_{\mathcal{P}} \Gamma / \mathcal{P}(k), M \#_{\mathcal{P}} \Gamma\right) .
$$

As an application, we obtain the isomorphism (1.11) in Corollary 6.5. Using this, the calculations of [24] compute $H C_{0}\left(\Gamma^{\infty}: I_{S}\right)$ for $S \in\left\{\ell^{p}, \ell^{ \pm p}\right\}$ (Lemma 6.9). Theorem 6.11 shows that if $k$ is a field and $R$ is unital then there is a quasiisomorphism

$$
\mathbb{H}(\Gamma / \mathcal{P}, H C(R / \mathcal{P}(k))) \stackrel{\sim}{\longrightarrow} H C\left(R \#_{\mathcal{P}} \Gamma / k\right) .
$$

The identity (1.9) follows from this (Corollary 6.6). In the particular case when $R$ is a commutative $\mathbb{Q}$-algebra, we obtain (in Subsection 6.7) a Hodge decomposition

$$
H C_{n}\left(R \#_{\mathcal{P}} \Gamma\right)=\bigoplus_{p=0}^{n} H C_{n}^{(p)}\left(R \#_{\mathcal{P}} \Gamma\right)=\bigoplus_{p=0}^{n} \mathbb{H}_{n}\left(\Gamma / \mathcal{P}: H C^{(p)}(R / \mathcal{P})\right) .
$$

The decomposition (1.12) follows from this. In Section 7 we study the groups $H C_{*}\left(\Gamma^{\infty}(\mathfrak{A}): I_{S(\mathfrak{A})}\right)$. The identities (1.5) and (1.6) are proved in Proposition 7.3. Theorem 7.5 proves that the comparison map (1.3) is an isomorphism when $\mathfrak{A}$ is a $C^{*}$-algebra and that (1.4) is an isomorphism when $\mathfrak{A}$ is a unital Banach algebra. The 
identity (1.13) is proved in Theorem 7.7. The latter is deduced from a computation of $H C_{*}^{(p)}\left(\ell^{\infty} / S\right)$ (Theorem 7.6) which, we think, is of independent interest. The identity (1.14) is included in Proposition 7.12, which considers also the case when $p \notin \mathbb{Z}$ and computes some of the groups $H C_{n}^{(q)}\left(\Gamma^{\infty}: I_{\ell^{ \pm p}}\right)$.

Acknowledgments. This article is part of an ongoing joint research project with Beatriz Abadie. It was originally part of our joint paper [1], which we later decided to split into two articles, to facilitate publication. Although she had important contributions to the present article-particularly to Section 3-she insisted in not being included as an author. I am indebted to her as well as to the Universidad de la República for its hospitality during my many visits to Beatriz to collaborate in this project over the last five years.

\section{Preliminaries}

2.1. Symmetric sequence ideals and the algebra $\Gamma^{\infty}(\mathfrak{A})$. Throughout this paper we work in the setting of bornological spaces and bornological algebras; a quick introduction to the subject is given in [12, Chapter 2]. Recall that a (complete, convex) bornological vector space over the field $\mathbb{C}$ of complex numbers is a filtering union $\mathbb{V}=\cup_{D} \mathbb{V}_{D}$ of Banach spaces, indexed by the disks of $\mathbb{V}$, such that the inclusions $\mathbb{V}_{D} \subset \mathbb{V}_{D^{\prime}}$ are bounded. A subset of $\mathbb{V}$ is bounded if it is a bounded subset of some $\mathbb{V}_{D}$. Let $X$ be a nonempty set. A map $X \rightarrow V$ is bounded if its image is contained in a bounded subset. We write $\ell^{\infty}(X, \mathbb{V})$ for the bornological vector space of bounded maps $X \rightarrow \mathbb{V}$ where $B \subset \ell^{\infty}(X, \mathbb{V})$ is bounded if $\bigcup_{b \in B} b(X)$ is. The inverse monoid $\operatorname{Emb}(X)$ of partially defined embeddings $X \rightarrow X$ acts on $\ell^{\infty}(X, \mathbb{V})$ by means of the following action

$$
\left(f_{*}(\alpha)_{x}= \begin{cases}\alpha_{f^{\dagger}(x)} & \text { if } x \in \operatorname{ran}(f) \\ 0 & \text { otherwise. }\end{cases}\right.
$$

When $X=\mathbb{N}$ or $\mathbb{V}=\mathbb{C}$, we omit it from our notation; thus $\operatorname{Emb}=\operatorname{Emb}(\mathbb{N})$, $\ell^{\infty}(\mathbb{V})=\ell^{\infty}(\mathbb{N}, \mathbb{V}), \ell^{\infty}(X)=\ell^{\infty}(X, \mathbb{C})$ and $\ell^{\infty}=\ell^{\infty}(\mathbb{N}, \mathbb{C})$. A subspace $S \triangleleft \ell^{\infty}$ is called symmetric if it is stable under the action of Emb. If $S \subset \ell^{\infty}$ is a symmetric subspace and $\mathbb{V}$ is a bornological vector space, then

$$
S(\mathbb{V}):=\left\{\alpha \in \ell^{\infty}(\mathbb{V}):(\exists D) \alpha(\mathbb{N}) \subset \mathbb{V}_{D} \text { and }\|\alpha\|_{D} \in S\right\}
$$

is a symmetric subspace of $\ell^{\infty}(\mathbb{V})$.

We will often work with sequences indexed by infinite countable sets other than $\mathbb{N}$. A bijection $u: \mathbb{N} \rightarrow X$ gives rise to a bounded isomorphism $\alpha \mapsto$ $\alpha u$ between $\ell^{\infty}(X, \mathbb{V})$ and $\ell^{\infty}(\mathbb{V})$. If $S \subset \ell^{\infty}$ is a symmetric subspace, we 
define $S(X, \mathbb{V})=\left\{s u^{-1}: s \in S(\mathbb{V})\right\}$. Because $S$ is symmetric by assumption, this definition does not depend on the choice of $u$.

Recall a bornological algebra is a bornological vector space $\mathfrak{A}$ with an associative bounded multiplication. If $\mathfrak{A}$ is a bornological algebra, then pointwise multiplication makes $\ell^{\infty}(\mathfrak{A})$ into a bornological algebra, and if $S \triangleleft \ell^{\infty}$ is a symmetric ideal, then $S(\mathfrak{A}) \triangleleft \ell^{\infty}(\mathfrak{A})$ is a symmetric two-sided ideal.

Let $R$ be a ring and $A: \mathbb{N} \times \mathbb{N} \rightarrow R$ a countably infinite square matrix with entries in $R$. For $i, j \in \mathbb{N}$, consider the following elements of $\mathbb{Z} \cup\{\infty\}$ :

$$
\begin{gathered}
r_{i}(A)=\#\left\{j: A_{i j} \neq 0\right\}, c_{j}(A)=\#\left\{i: A_{i j} \neq 0\right\}, \\
N(A):=\sup \left\{r_{i}(A), c_{i}(A): i \in \mathbb{N}\right\} .
\end{gathered}
$$

Let $\mathfrak{A}$ be a bornological algebra, and $S \triangleleft \ell^{\infty}(\mathfrak{A})$ an ideal. Following [1, Definition 3.5], we set

$$
\begin{gathered}
I_{S(\mathfrak{A})}=\left\{A=\left(A_{i j}\right)_{i, j \in \mathbb{N}}:\left\{A_{i j}\right\} \in S(\mathbb{N} \times \mathbb{N}) \text { and } N(A)<\infty\right\} \\
\text { and } \Gamma^{\infty}(\mathfrak{A})=I_{\ell \infty(\mathfrak{A}) .}
\end{gathered}
$$

2.2. Crossed products with $\Gamma$. Let $R$ be a ring. Karoubi's cone of the ring $R$ is the ring

$$
\Gamma(R)=\left\{A \in M_{\mathbb{N}}(R): N(A)<\infty \text { and } \#\left\{A_{i, j}:(i, j) \in \mathbb{N} \times \mathbb{N}\right\}<\infty\right\} .
$$

We also consider the ring of all locally constant sequences

$$
\mathcal{P}(R)=\left\{\alpha \in R^{\mathbb{N}}: \#\left\{\alpha_{n}: n \in \mathbb{N}\right\}<\infty\right\} .
$$

Observe that $\alpha \in \mathcal{P}(R)$ if and only if the diagonal matrix $\operatorname{diag}(\alpha) \in \Gamma(R)$. We shall identify $\mathcal{P}(R)$ with $\operatorname{diag}(\mathcal{P}(R)) \subset \Gamma(R)$. When $R=\mathbb{Z}$ we omit it from our notation; we set

$$
\Gamma=\Gamma(\mathbb{Z}), \quad \mathcal{P}=\mathcal{P}(\mathbb{Z}) .
$$

By [8, Lemma 4.7.1] the map

$$
\phi: \Gamma \otimes R \rightarrow \Gamma(R), \quad \phi(A \otimes x)_{i, j}=A_{i, j} x
$$

is an isomorphism. It follows from this that $\Gamma$ and $\mathcal{P}$ are flat $\mathbb{Z}$-modules. By [1, Remark 6.8] the restriction of $\phi$ induces an isomorphism

$$
\mathcal{P} \otimes R \stackrel{\cong}{\longrightarrow} \mathcal{P}(R) \text {. }
$$

There is a monoid homomorphism

$$
U: \mathrm{Emb} \rightarrow \Gamma, \quad\left(U_{f}\right)_{i, j}=\left\{\begin{array}{cc}
1 & \text { if } j \in \operatorname{dom}(f) \text { and } f(j)=i \\
0 & \text { otherwise }
\end{array}\right.
$$


Observe that the idempotent submonoid of Emb is isomorphic to the monoid $2^{\mathbb{N}}$ of subsets of $\mathbb{N}$ with intersection of subsets as multiplication. If $p^{2}=p$ and $A=\operatorname{Im} p$, then $U_{p}=\operatorname{diag}\left(\chi_{A}\right)$ is a diagonal matrix. We will often identify $p, U_{p}$ and $\chi_{A}$. We also consider the monoid rings $\mathbb{Z}\left[2^{\mathbb{N}}\right]$ and $\mathbb{Z}[\mathrm{Emb}]$, and the two-sided ideals

$$
\begin{gathered}
I=\left\langle\left\{\chi_{A \sqcup B}-\chi_{A}-\chi_{B}: A, B \subset \mathbb{N}, A \cap B=\emptyset\right\}\right\rangle \triangleleft \mathbb{Z}\left[2^{\mathbb{N}}\right], \\
J=\left\langle\left\{\chi_{A \sqcup B}-\chi_{A}-\chi_{B}: A, B \subset \mathbb{N}, \quad A \cap B=\emptyset\right\}\right\rangle \triangleleft \mathbb{Z}[\text { Emb }] .
\end{gathered}
$$

The following lemma follows from [1, Lemma 5.4 and Remark 6.8].

Lemma 2.1. Let $R$ be a ring. The maps (2.2.3), (2.2.1) and (2.2.2) induce the following isomorphisms:

i) $\mathcal{P}(R)=R\left[2^{\mathbb{N}}\right] / R \otimes I$.

ii) $\Gamma(R)=R[\mathrm{Emb}] / R \otimes J$.

Remark 2.2. Given a monoid $M$ and a unital ring $R$, a representation of $M$ in $R$ modules is the same thing as a module over the monoid algebra $R[M]$. In view of Lemma 2.1, the modules over $\mathcal{P}(R)$ and $\Gamma(R)$ correspond to those representations of the inverse monoids $2^{\mathbb{N}}$ and Emb which are tight in the sense of Exel (see [14, Def. 13.1 and Prop. 11.9]).

Because Emb is a monoid, if $\mathcal{A}$ is a ring on which Emb acts by algebra endomorphisms we can form the crossed product $\mathcal{A} \#$ Emb. As an abelian group, $\mathcal{A} \#$ Emb $=\mathcal{A} \otimes_{\mathbb{Z}} \mathbb{Z}[\mathrm{Emb}]$ with multiplication given by

$$
(a \# f)(b \# g)=a f_{*}(b) \# f g .
$$

Here $\#=\otimes$ and $f_{*}(b)$ denotes the action of $f$ on Emb. Now assume that the Embring $\mathcal{A}$ is also a $\mathcal{P}$-algebra, that is, it is a ring and a $\mathcal{P}$-bimodule, and these operations are compatible in the sense that

$$
(a p) b=a(p b) \quad(a, b \in \mathcal{A}, \quad p \in \mathcal{P}) .
$$

Further assume that $\mathcal{A}$ is central as a $\mathcal{P}$-bimodule, i.e. $p a=a p(a \in \mathcal{A}, p \in \mathcal{P})$, and that

$$
p a=p_{*}(a) \quad\left(p \in 2^{\mathbb{N}}\right) .
$$

Under all these conditions, we say that $\mathcal{A}$ is an Emb-bundle (cf. [2, Def. 2.1]). For $J \triangleleft \mathbb{Z}[\mathrm{Emb}]$ as in (2.2.5), we have

$$
\begin{gathered}
\mathcal{A} \# \text { Emb } \triangleright \mathcal{A} \# J=\operatorname{span}\{r \# j: r \in \mathcal{A}, j \in J\} \text { and } \\
\mathcal{A} \# \text { Emb } \triangleright L=\operatorname{span}\{r p \# h-r \# p h: r \in \mathcal{A}, p \in \mathcal{P}, h \in \mathrm{Emb}\} .
\end{gathered}
$$

Set

$$
\mathcal{A} \#_{\mathcal{P}} \Gamma=\mathcal{A} \# \mathrm{Emb} /(L+\mathcal{A} \# J) .
$$

Thus, $\mathcal{A} \#_{\mathcal{P}} \Gamma=\mathcal{A} \otimes_{\mathcal{P}} \Gamma$ as left $\mathcal{P}$-modules, and the product is that induced by (2.2.6); we have

$$
\left(a \# U_{f}\right)\left(b \# U_{g}\right)=a f_{*}(b) \# U_{f g} \in \mathcal{A} \# \#_{\mathcal{P}} \Gamma .
$$


Proposition 2.3. ([1, Proposition 6.11]) Let $\mathfrak{A}$ be a bornological algebra. The map

$$
\ell^{\infty}(\mathfrak{A}) \#_{\mathcal{P}} \Gamma \rightarrow \Gamma^{\infty}(\mathfrak{A}), \quad \alpha \# U_{f} \mapsto \operatorname{diag}(\alpha) U_{f}
$$

is an isomorphism of $\mathcal{P}$-algebras. If $S \triangleleft \ell^{\infty}$ is a symmetric ideal, then (2.2.9) sends $S(\mathfrak{A}) \#_{\mathcal{P}} \Gamma$ isomorphically onto $I_{S(\mathfrak{A})} \triangleleft \Gamma^{\infty}(\mathfrak{A})$.

\section{Flat ideals of $\Gamma^{\infty}$ and $\ell^{\infty}$}

Proposition 3.1. Every finitely generated ideal of $\ell^{\infty}$ is principal and projective.

Proof. The fact that the finitely generated ideals of $\ell^{\infty}$ are projective follows from [18, Corollary 2.4]. We will prove that they are principal. Given $\alpha \in \ell^{\infty}$, set

$$
v_{\alpha}(n)= \begin{cases}0, & \text { if } \alpha(n)=0 \\ \frac{\alpha(n)}{|\alpha(n)|}, & \text { otherwise }\end{cases}
$$

Notice that $v_{\alpha}$ is the partial isometry in the polar decomposition of $\alpha$. In fact, we have

$$
\alpha=v_{\alpha}|\alpha|, \quad|\alpha|=\bar{v}_{\alpha} \alpha .
$$

It follows that, for any ideal $I$ in $\ell^{\infty}, \alpha \in I$ if and only if $|\alpha| \in I$. Now let $I$ be an ideal of $\ell^{\infty}$ generated by $\left\{\alpha_{0}, \alpha_{1}\right\}$, and set

$$
\mu(n)=\max \left\{\left|\alpha_{0}(n)\right|,\left|\alpha_{1}(n)\right|\right\} .
$$

For $i=0,1$, let

$$
\gamma_{i}(n)=\left\{\begin{array}{cc}
1 / 2 & \text { if }\left|\alpha_{0}(n)\right|=\left|\alpha_{1}(n)\right| \\
1 & \text { if }\left|\alpha_{i}(n)\right|>\left|\alpha_{1-i}(n)\right| \\
0 & \text { otherwise. }
\end{array}\right.
$$

We have $\mu=\gamma_{0}\left|\alpha_{0}\right|+\gamma_{1}\left|\alpha_{1}\right|$; thus $\mu \in I$. Now set

$$
\tau_{i}(n)= \begin{cases}0 & \text { if } \mu(n)=0 \\ \frac{\alpha_{i}(n)}{\mu(n)} & \text { otherwise. }\end{cases}
$$

Then $\alpha_{i}=\tau_{i} \mu,(i=0,1)$. Notice that $\tau_{i} \in \ell^{\infty}$, since $\left|\tau_{i}(n)\right| \leq 1$ for all $n \in \mathbb{N}$, $i=0,1$. Therefore, $\mu$ generates $I$. The general case can now be proven by induction on the number of generators.

Corollary. Every ideal of $\ell^{\infty}$ is flat.

Proposition 3.2. Let $\mathfrak{A}$ be a unital Banach algebra and $S \triangleleft \ell^{\infty}$ a symmetric ideal. Assume that

$$
\alpha \in S \Rightarrow \sqrt{|\alpha|} \in S .
$$

Then $S(\mathfrak{A}) \triangleleft \ell^{\infty}(\mathfrak{A})$ is flat both as a right and as a left $\ell^{\infty}(\mathfrak{A})$-module. 
Proof. Consider the following homomorphism of $\ell^{\infty}(\mathfrak{A})$-modules

$$
\mu: \ell^{\infty}(\mathfrak{A}) \otimes_{\ell} S \rightarrow S(\mathfrak{A}), \quad \mu(\alpha \otimes \beta)_{n}=\alpha_{n} \beta_{n} .
$$

We claim that $\mu$ is an isomorphism. To prove it is surjective, for $\alpha \in S(\mathfrak{A})$ let $v_{\alpha}$ be as in (3.1). Then $v_{\alpha} \in \ell^{\infty}(\mathfrak{A})$ and

$$
\alpha=\mu\left(v_{\alpha} \otimes\|\alpha\|\right) .
$$

Thus $\mu$ is surjective. To prove it is also injective, let

$$
\eta=\sum_{i=1}^{n} \alpha^{i} \otimes \beta^{i} \in \operatorname{ker} \mu .
$$

By Proposition 3.1, the ideal $\left\langle\beta^{1}, \ldots, \beta^{n}\right\rangle \triangleleft \ell^{\infty}$ is principal. Let $\beta$ be a generator; we may and do choose it so that $\beta=|\beta|$. By bilinearity, we may rewrite $\eta$ as a single elementary tensor and we have

$$
\eta=\alpha \otimes \beta, \alpha \beta=0
$$

But $\alpha \beta=0$ implies $\alpha \sqrt{\beta}=0$, whence

$$
\eta=\alpha \sqrt{\beta} \otimes \sqrt{\beta}=0 .
$$

Thus the claim is proved. It follows that $S(\mathfrak{A})$ is flat as a left $\ell^{\infty}(\mathfrak{A})$-module, since it is the scalar extension of $S$, which is a flat $\ell^{\infty}$-module by Corollary 3 . The proof that $S(\mathfrak{A})$ is flat on the right is similar.

Examples 3.2. The hypothesis of Proposition 3.2 are satisfied, for example, when $S$ is either of $\ell^{\infty-}, c_{0}$.

Proposition 3.3. Every two-sided ideal of $\Gamma^{\infty}$ is flat both as a left and as a right $\Gamma^{\infty}$-module.

Proof. Let $I \triangleleft \Gamma^{\infty}$. By [1, Theorem 4.5] there is a symmetric ideal $S$ such that $I=I_{S}$. Observe that

$$
I_{S}=S \otimes_{\mathcal{P}} \Gamma=S \otimes_{\ell} \ell^{\infty} \otimes_{\mathcal{P}} \Gamma=S \otimes_{\ell} \Gamma^{\infty} .
$$

Thus $I_{S} \otimes_{\Gamma \infty}=S \otimes_{\ell}$ is exact by Corollary 3 . Hence $I$ is flat as a right module and therefore also as a left module, since $\Gamma^{\infty}$ is a $*$-algebra.

Remark 3.4. By [1, Proposition 4.6], if $k$ is a field, then $M_{\infty} k$ is the only proper two-sided ideal of $\Gamma(k)$. Observe that $M_{\infty} k$ is projective both as a left and as a right module, since it is isomorphic to an infinite sum of copies of the principal ideal generated by the idempotent $E_{1,1}$. 
Proposition 3.5. Let $\mathfrak{A}$ be a unital Banach algebra and $S \triangleleft \ell^{\infty}$ a symmetric ideal as in Proposition 3.2. Then $I_{S(\mathfrak{A})}$ is flat both as a left and as a right $\Gamma^{\infty}(\mathfrak{A})$-module.

Proof. By Proposition 2.3 and the proof of Proposition 3.2 we have the following canonical isomorphisms of right $\Gamma^{\infty}(\mathfrak{A})$-modules

$$
I_{S(\mathfrak{A})}=S(\mathfrak{A}) \otimes_{\mathcal{P}} \Gamma=S \otimes_{\ell} \ell^{\infty}(\mathfrak{A}) \otimes_{\mathcal{P}} \Gamma=S \otimes_{\ell} \Gamma^{\infty}(\mathfrak{A}) .
$$

This, together with Corollary 3 , proves that $I_{S(\mathfrak{A})}$ is flat as a right $\Gamma^{\infty}(\mathfrak{A})$-module. The proof that it is also flat on the left is similar.

\section{Flatness properties of $\mathcal{P}$}

Let $k$ be a commutative ring. Recall that a $k$-algebra $A$ which is projective as an $A \otimes_{k} A^{o p}$-module is called separable.

Proposition 4.1. The $k$-algebra $\mathcal{P}(k)$ is a filtering union of separable algebras.

Proof. We shall show that $\mathcal{P}$ is a filtering union of finite products of copies of $\mathbb{Z}$, indexed by the finite partitions of $\mathbb{N}$. Here a finite partition of $\mathbb{N}$ is a finite set $\pi=\left\{A_{1}, \ldots, A_{n}\right\}$ of subsets of $\mathbb{N}$ such that $\mathbb{N}=A_{1} \sqcup \cdots \sqcup A_{n}$. We say that a partition $\rho=\left\{B_{1}, \ldots, B_{m}\right\}$ is finer than $\pi$ if the following condition is satisfied:

$$
(\forall 1 \leq i \leq m)(\exists j) \quad B_{i} \subset A_{j} .
$$

Note that if $\pi$ and $\pi^{\prime}$ are any two finite partitions, then

$$
\pi \wedge \pi^{\prime}=\left\{B \subset \mathbb{N}:\left(\exists A \in \pi, A^{\prime} \in \pi^{\prime}\right) B=A \cap A^{\prime}\right\} .
$$

is a finite partition and is finer than each of them. Thus the set

$$
\operatorname{Part}(\mathbb{N})=\{\pi \text { finite partition of } \mathbb{N}\} .
$$

is a filtered partially ordered set. If $\pi \in \operatorname{Part}(\mathbb{N})$ has $n$ elements, put

$$
\mathcal{P} \supset R_{\pi}=\bigoplus_{i=1}^{n} \mathbb{Z} P_{A_{i}} .
$$

Observe that $R_{\pi} \cong \mathbb{Z}^{n}$ and that $\mathcal{P}=\bigcup_{\pi} R_{\pi}$. This proves the proposition in the case $k=\mathbb{Z}$. The general case follows from this using the isomorphism $\mathcal{P} \otimes k \stackrel{\cong}{\longrightarrow} \mathcal{P}(k)$.

Corollary. If $k$ is a field, then $\mathcal{P}(k)$ is a von Neumann regular ring. In other words, every $\mathcal{P}(k)$-module is flat. 
Proposition 4.2. Let $R$ be a unital ring. Then $\Gamma(R)$ is flat, both as a left and as a right $\mathcal{P}(R)$-module.

Proof. We prove that $\Gamma(R)$ is flat as a right $\mathcal{P}(R)$-module; the proof that it is also flat on the left is similar. If $M$ is a $\mathcal{P}(R)$-module, then

$$
\Gamma(R) \otimes_{\mathcal{P}(R)} M=\Gamma \otimes R \otimes_{\mathcal{P} \otimes R} M=\Gamma \otimes_{\mathcal{P}} M .
$$

Hence it suffices to consider the case $R=\mathbb{Z}$. In view of Proposition 4.1 and its proof, we have

$$
\Gamma \otimes_{\mathcal{P}} M=\operatorname{colim}_{\pi \in \operatorname{Part}(\mathbb{N})} \Gamma \otimes_{R_{\pi}} M .
$$

Hence it suffices to show that $\Gamma$ is flat as a module over $R_{\pi}$, for each $\pi \in \operatorname{Part}(\mathbb{N})$. We have

$$
R_{\pi}=\bigoplus_{A \in \pi} \mathbb{Z} P_{A}
$$

Hence

$$
\Gamma \otimes_{R_{\pi}} M=\bigoplus_{A \in \pi} \Gamma p_{A} \otimes p_{A} M .
$$

Thus it suffices to show that $\Gamma p_{A}$ is flat as an abelian group. Since $\Gamma p_{A}$ is a direct summand of $\Gamma$, we are reduced to showing that $\Gamma$ is $\mathbb{Z}$-flat. As said above, the map (2.2.1) is an isomorphism for every ring; in particular this applies to show that if $M$ is any abelian group-regarded as a ring with trivial multiplication-then $\Gamma \otimes M=\Gamma(M)$. Since $M \rightarrow \Gamma(M)$ is clearly exact, this conlcudes the proof.

\section{Excision}

A ring $A$ is called $K$-excisive if for every ideal embedding $A \triangleleft B$ the map $K_{*}(A) \rightarrow K_{*}(B: A)$ is an isomorphism. It was proved by Suslin and Wodzicki [20, Theorem $C$ ] that if a ring $A$ satisfies the following property then it is $K$-excisive.

$$
\begin{gathered}
\forall n, \forall a \in A^{\oplus n}, \exists b \in A^{\oplus n}, \quad c, d \in A \text {, such that } a=c d b \text { and such that } \\
\left(0:_{A} d\right)_{r}:=\{v \in A: d v=0\}=\left(0:_{A} c d\right)_{r} .
\end{gathered}
$$

The right ideal $\left(0::_{A} d\right)_{r}$ is called the right annihilator of $d$ in $A$. The property above is the so-called left triple factorization property (TFP). A ring is $K$-excisive if and only if its opposite ring $A^{o p}$ is ([20, Remark (1) pp 53]), so rings satisfying the right TFP are excisive also. Further results of Wodzicki ([23, Theorems 1.1 and 3.1]) and of Suslin-Wodzicki ([20, Theorem B]) establish that a $\mathbb{Q}$-algebra $A$ is 
$K$-excisive if and only if it is excisive for cyclic homology, and that this happens if and only if the bar complex $\left(C_{*}^{b a r}(A), b^{\prime}\right)$ is exact. Here

$$
\begin{gathered}
b^{\prime}: C_{n+1}^{\text {bar }}(A)=A^{\otimes n+2} \rightarrow A^{\otimes n+1}=C_{n}^{b a r}(A) \quad(n \geq 0) \\
b^{\prime}\left(a_{0} \otimes \cdots \otimes a_{n+1}\right)=\sum_{i=0}^{n}(-1)^{i} a_{0} \otimes \cdots \otimes a_{i} a_{i+1} \otimes \cdots \otimes a_{n+1} .
\end{gathered}
$$

The tensor products above are taken over $\mathbb{Z}$ or, equivalently, over $\mathbb{Q}$, since $A$ is assumed to be a $\mathbb{Q}$-algebra. The $\mathbb{Q}$-algebras whose bar homology vanishes-that is, the $K$-excisive ones-are also called $H$-unital.

Proposition 5.1. Let $\mathfrak{A}$ be a bornological algebra and $S \triangleleft \ell^{\infty}$ a symmetric ideal. Assume that $S(\mathfrak{A})$ has the (left or right) triple factorization property. Then $I_{S(\mathfrak{A})}$ is $K$-excisive.

Proof. Assume that $S(\mathfrak{A})$ has the left TFP. We have to prove that $I_{S(\mathfrak{A})}$ is $H$-unital. Let $n \geq 0$ and let $z \in C_{n}^{b a r}\left(I_{S(\mathfrak{A})}\right)$ be a cycle. We may write

$$
z=\sum_{i=1}^{m} \operatorname{diag}\left(\alpha^{0, i}\right) U_{f_{0, i}} \otimes \cdots \otimes \operatorname{diag}\left(\alpha^{n, i}\right) U_{f_{n, i}},
$$

where $\operatorname{supp}\left(\alpha^{j, i}\right)=\operatorname{ran}\left(f_{j, i}\right)$ for all $i, j$. By TFP, there are elements $\gamma, \delta$ and $\beta^{1}, \ldots, \beta^{m}$ in $S(\mathfrak{A})$ such that $\alpha^{0, i}=\gamma \delta \beta^{i}(1 \leq i \leq m)$, and such that

$$
(0: S(\mathfrak{A}) \gamma \delta)_{r}=(0: S(\mathfrak{A}) \delta)_{r} .
$$

Now observe that if $\theta \in S(\mathfrak{A})$ then, by our definition of $I_{S(\mathfrak{A})}$ (2.1.1), we have

$$
\left(0:_{I_{S(\mathfrak{A})}} \operatorname{diag}(\theta)\right)_{r}=\left\{T \in I_{S(\mathfrak{A})}:(\forall j) T_{*, j} \in\left(0:_{S(\mathfrak{A})} \theta\right)_{r}\right\} .
$$

Hence, (5.1) implies that

$$
\left(0:_{I_{S(\mathfrak{A})}} \operatorname{diag}(\gamma \delta)\right)_{r}=\left(0:_{I_{S(\mathfrak{L})}} \operatorname{diag}(\delta)\right)_{r} .
$$

Put

$$
y=\sum_{i} \operatorname{diag}\left(\beta^{i}\right) U_{f_{0, i}} \otimes \operatorname{diag}\left(\alpha^{1, i}\right) U_{f_{1, i}} \otimes \cdots \otimes \operatorname{diag}\left(\alpha^{n, i}\right) U_{f_{n, i}} .
$$

Consider the following element of $C_{n+1}^{b a r}\left(I_{S(\mathfrak{A})}\right)$

$$
w=\operatorname{diag}(\gamma) \otimes \operatorname{diag}(\delta) y .
$$

We have

$$
b^{\prime}(w)=z-\operatorname{diag}(\gamma) \otimes \operatorname{diag}(\delta) b^{\prime}(y) .
$$


If $n=0$ then $b^{\prime}(y)=0$, so this proves that $z$ is a boundary. We have to show that $\operatorname{diag}(\delta) b^{\prime}(y)=0$ if $n \geq 1$. Choose a basis $\left\{v_{l}\right\}$ of the $\mathbb{Q}$-vector space $C_{n-1}^{b a r}\left(I_{S(\mathfrak{A})}\right)$. Then $y=\sum_{l} T_{l} \otimes v_{l}$ for unique $T_{l} \in I_{S(\mathfrak{R})}$, and

$$
0=b^{\prime}(z)=\operatorname{diag}(\gamma \delta) b^{\prime}(y)=\sum_{l} \operatorname{diag}(\gamma \delta) T_{l} \otimes v_{l} .
$$

Hence we must have $\operatorname{diag}(\gamma \delta) T_{l}=0$ for all $l$, and therefore $\operatorname{diag}(\delta) b^{\prime}(y)=0$ by (5.2).

Example 5.2. Any Banach algebra with a bounded left approximate unit satisfies the Cohen-Hewitt factorization property; thus it has the left TFP ([6, Lemma 6.5.1]). In particular, this applies to $C^{*}$-algebras. If $\mathfrak{A}$ is a $C^{*}$-algebra then $c_{0}(\mathfrak{A})$ is again a $C^{*}$-algebra; hence $I_{c_{0}(\mathfrak{A})}$ is $K$-excisive, by Proposition 5.1.

Example 5.3. If $\mathfrak{A}$ is a unital Banach algebra then $\ell^{\infty-}(\mathfrak{A})$ has the TFP. To see this, let $\alpha^{1}, \ldots, \alpha^{m} \in \ell^{\infty-}$. Choose $p$ such that $\alpha^{i} \in \ell^{p}(\mathfrak{A})$ for all $i$. For each $n$ put

$$
\gamma_{n}=\max _{1 \leq i \leq m}\left\|\alpha_{n}^{i}\right\|, \quad \beta_{n}^{i}=\left\{\begin{array}{cc}
\alpha_{n}^{i} / \gamma_{n}^{1 / 2} & \text { if } \gamma_{n} \neq 0 \\
0 & \text { otherwise. }
\end{array}\right.
$$

Then $\left\|\beta_{n}^{i}\right\| \leq\left\|\alpha_{n}^{i}\right\|^{1 / 2}$ and therefore $\beta^{i} \in \ell^{2 p}(\mathfrak{A})$. Similarly $\gamma^{1 / 4} \in \ell^{4 p}(\mathfrak{A})$. One checks that the factorization $\alpha^{i}=\gamma^{1 / 4} \gamma^{1 / 4} \beta^{i}$ satisfies the requirements of the TFP.

\section{Homology of crossed products with $\Gamma$}

6.1. Homology of augmented algebras. In this subsection $A$ and $B$ will be unital rings; furthermore, $B$ will be an $A$-algebra, that is, $B$ will be a ring together with a unital ring homomorphism $\iota: A \rightarrow B$. Further assume that $A$ is equipped with a left $B$-module structure and a surjective $B$-module homomorphism $\pi: B \rightarrow A$ such that $\pi \iota=i d_{A}$. Observe that the triple $(B, A, \pi)$ is an augmented ring in the sense of Cartan-Eilenberg [4, Chapter VIII, $\$ 1]$. Since in addition, $B$ is an $A$-algebra, we call the triple $(B, A, \pi)$ an augmented algebra. Let $M$ be a right $B$-module. Consider the simplicial $A$-module $\perp(B / A, M)$ given in dimension $n$ by

$$
\perp_{n}(B / A, M)=M \otimes_{A} B^{\otimes_{A} n},
$$

with face and degeneracy maps defined as follows $(n \geq 0)$

$$
\begin{gathered}
\partial_{i}: \perp_{n+1}(B / A, M) \rightarrow \perp_{n}(B / A, M), \\
\partial_{i}\left(x_{0} \otimes \cdots \otimes x_{n+1}\right)=\left\{\begin{array}{cc}
x_{0} \otimes \cdots \otimes x_{i} x_{i+1} \otimes \cdots \otimes x_{n+1} & i \leq n \\
x_{0} \otimes \cdots \otimes x_{n} \pi\left(x_{n+1}\right) & i=n+1
\end{array}\right. \\
\delta_{i}: \perp_{n}(B / A, M) \rightarrow \perp_{n+1}(B / A, M), \quad(0 \leq i \leq n) \\
\delta_{i}\left(x_{0} \otimes \cdots \otimes x_{n}\right)=x_{0} \otimes \cdots \otimes x_{i} \otimes 1 \otimes x_{i+1} \otimes \cdots \otimes x_{n} .
\end{gathered}
$$


The homology of $(B / A, M)$ relative to $(A, B, \pi)$, denoted $H_{*}(B / A, M)$, is the homotopy of the simplicial module $\perp(B / A, M)$;

$$
H_{*}(B / A, M)=\pi_{*}(\perp(B / A, M))=H_{*}(\perp(B / A, M), \partial) .
$$

Here

$$
\partial=\sum_{i=0}^{n+1}(-1)^{i} \partial_{i}: \perp_{n+1}(B / A, M) \rightarrow \perp_{n}(B / A, M)
$$

is the alternating sum of the face maps. We have

$$
H_{0}(B / A, M)=M \otimes_{B} A .
$$

Let $P(B / A)=\perp(B / A, B) ; \pi: P(B / A) \rightarrow A$ is a resolution which is projective relative to $B / A$, and $\perp(B / A, N)=N \otimes_{B} P(B / A)$. Hence if $B$ is flat both as a left and as a right $A$-module, then

$$
H_{*}(B / A, M)=\operatorname{Tor}_{*}^{B}(M, A) .
$$

Without flatness assumptions, we may regard the groups $H_{*}(B / A, M)$ as relative Tor groups.

Lemma 6.1. Let $N$ be a right $B$-module. Consider $N^{2}=N^{1 \times 2}$ as a right module over $M_{2} B$ via the matrix product. View $M_{2} B$ as an $A \oplus A$-algebra through the diagonal embedding $\left(a_{1}, a_{2}\right) \mapsto E_{11} a_{1}+E_{22} a_{2}$. Then the map

$$
\begin{gathered}
\iota: \perp(B / A, N) \rightarrow \perp\left(M_{2}(B) / A \oplus A, N \oplus N\right) \\
\iota\left(x_{0} \otimes \cdots \otimes x_{n}\right)=E_{11} x_{0} \otimes \cdots \otimes E_{11} x_{n}
\end{gathered}
$$

is a quasi-isomorphism.

Proof. Consider the maps

$$
\begin{gathered}
\iota^{\prime}: P(B / A)^{2 \times 1} \rightarrow P\left(M_{2} B / A^{2}\right), \\
\iota^{\prime}\left(E_{i 1}\left(x_{0} \otimes \cdots \otimes x_{n}\right)\right)=E_{i 1} x_{0} \otimes E_{11} x_{1} \otimes \cdots \otimes E_{11} x_{n}, \\
\text { and } p^{\prime}: P\left(M_{2} B / A^{2}\right) \rightarrow P(B / A)^{2 \times 1}, \\
p^{\prime}\left(E_{i_{0}, i_{1}} x_{0} \otimes \cdots \otimes E_{i_{n}, i_{n+1}} x_{n}\right)=E_{i_{0} 1}\left(x_{0} \otimes \cdots \otimes x_{n}\right) .
\end{gathered}
$$

One checks that both $\iota^{\prime}$ and $p^{\prime}$ are $M_{2} B$-linear chain homomorphisms, and that $p^{\prime} \iota^{\prime}=1$. In particular $\pi^{2 \times 1}: P(B / A)^{2 \times 1} \rightarrow A^{2 \times 1}$ is a projective resolution relative to $M_{2} A / A^{2}$, whence

$$
\iota=N^{1 \times 2} \otimes_{M_{2} B} \iota^{\prime}
$$

is a quasi-isomorphism, as claimed. 
6.2. The augmented algebra $\left(\Gamma, \mathcal{P}, \epsilon_{l}\right)$. Regarding the elements of $2^{\mathbb{N}}$ as sequences of zeros and ones, there is an obvious action $\mathrm{Emb} \times 2^{\mathbb{N}} \rightarrow 2^{\mathbb{N}},(f, p) \mapsto f_{*}(p)$. It agrees with the inner action; we have

$$
f_{*}(p)=f p f^{\dagger} .
$$

Thus $\mathbb{Z}\left[2^{\mathbb{N}}\right]$ is a $\mathbb{Z}[\mathrm{Emb}]$-module. Note that, if $A, B \subset \mathbb{N}$ are disjoint, then for $I \subset \mathbb{Z}\left[2^{\mathbb{N}}\right]$ as in (2.2.4) and $q \in 2^{\mathbb{N}}$, we have

$$
\begin{aligned}
& f_{*}\left(\left(p_{A \sqcup B}-p_{A}-p_{B}\right) q\right) \\
& \quad=\left(p_{f((A \sqcup B) \cap \operatorname{dom}(f))}-p_{f(A \cap \operatorname{dom}(f))}-p_{f(B \cap \operatorname{dom}(f))}\right) f_{*}(q) \in I, \\
& \begin{aligned}
\left(f\left(p_{A \sqcup B}-p_{A}-p_{B}\right) g\right)_{*}(q) & =f_{*}\left(\left(p_{A \sqcup B}-p_{A}-p_{B}\right)_{*}\left(g_{*}(q)\right)\right) \\
& =f_{*}\left(\left(p_{A \sqcup B}-p_{A}-p_{B}\right) \cdot g_{*}(q)\right) \in I .
\end{aligned}
\end{aligned}
$$

Thus $\mathcal{P}$ is a $\Gamma$-module. Let $f \in \mathrm{Emb}$; put

$$
\epsilon_{l}(f)=p_{\operatorname{ran}(f)} \in 2^{\mathbb{N}} \ni \epsilon_{r}(f)=\epsilon_{l}\left(f^{\dagger}\right)=p_{\operatorname{dom}(f)} .
$$

Note that

$$
\epsilon_{l}(f g)(n)=p_{\operatorname{ran}(f g)}(n)=\left\{\begin{array}{cc}
1 & \text { if } n \in f(\operatorname{dom}(f) \cap \operatorname{ran}(g)) \\
0 & \text { otherwise }
\end{array}\right\}=f_{*}\left(\epsilon_{l}(g)\right)(n) .
$$

Thus the induced linear map $\epsilon_{l}: \mathbb{Z}[\mathrm{Emb}] \rightarrow \mathbb{Z}\left[2^{\mathbb{N}}\right]$ is a homomorphism of left $\mathbb{Z}[\mathrm{Emb}]$-modules. In particular, if $A, B \subset \mathbb{N}$ are disjoint, we have

$$
\epsilon_{l}\left(f\left(p_{A \sqcup B}-p_{A}-p_{B}\right) g\right)=f_{*}\left(p_{A \sqcup B}-p_{A}-p_{B}\right) \epsilon_{l}(g) \in I .
$$

Hence $\epsilon_{l}$ induces a homomorphism of left $\Gamma$-modules

$$
\epsilon_{l}: \Gamma \rightarrow \mathcal{P} \text {. }
$$

Observe that the canonical inclusion $\mathcal{P} \subset \Gamma$, which is an algebra homomorphism, but not a $\Gamma$-module homomorphism, is a section of $\epsilon_{l}$. Thus we are in the augmented algebra setting described above. Moreover $\Gamma$ is flat over $\mathcal{P}$, by Proposition 4.2. Hence

$$
H_{*}(\Gamma / \mathcal{P}, M)=\operatorname{Tor}_{*}^{\Gamma}(M, \mathcal{P}) .
$$

Observe also that if $k$ is any commutative ring and $M$ is a $\Gamma(k)$-module, then

$$
C(\Gamma / \mathcal{P}, M)=C(\Gamma(k) / \mathcal{P}(k), M) .
$$

In particular,

$$
H_{*}(\Gamma / \mathcal{P}, M)=H_{*}(\Gamma(k) / \mathcal{P}(k), M) .
$$


In the next lemma and below we consider the following submonoids of Emb

$$
\operatorname{Emb} \supset \mathcal{E}=\{f: \operatorname{dom} f=\mathbb{N}\} \supset \mathcal{E}^{*}=\{f \in \mathcal{E}: \operatorname{ran}(f)=\mathbb{N}\} .
$$

If $M$ is a $\Gamma$-module and $\mathfrak{S} \in\left\{\mathcal{E}, \mathcal{E}^{*}\right\}$ we write

$$
M_{\mathfrak{S}}=M / \operatorname{span}\left\{m-f_{*}(m): f \in \mathfrak{S}\right\} .
$$

Here the span is $\mathbb{Z}$-linear.

Lemma 6.2. The kernel of $\epsilon_{l}: \Gamma \rightarrow \mathcal{P}$ is generated, as a left $\mathcal{P}$-module, by the elements $U_{f}-1, f \in \mathcal{E}^{*}$.

Proof. Let $K=\operatorname{ker}\left(\epsilon_{l}\right)$. It is clear that $K$ is generated, as an abelian group, by the elements $U_{f}-p_{\operatorname{ran} f}, f \in \mathrm{Emb}$. Assume that $f \in \mathrm{Emb}$ but $f \notin \mathcal{E}^{*}$. We claim that we may choose a subset $A \subset \operatorname{dom}(f)$ such that $B=\mathbb{N} \backslash A$ is bijectable to $\mathbb{N} \backslash f(A)$, and such that $\mathbb{N} \backslash(\operatorname{dom} f \cap B)$ is bijectable to $\mathbb{N} \backslash f(\operatorname{dom} f \cap B)$. Indeed if $\mathbb{N} \backslash \operatorname{dom} f$ is already bijectable to $\mathbb{N} \backslash \operatorname{ran} f$, we may take $A=\operatorname{dom} f$. Otherwise $\operatorname{dom} f$ is infinite, so we may split it into two disjoint infinite pieces, and take $A$ to be one of them. Thus the claim is proved. For such $A$, there exist $g, h \in \mathcal{E}^{*}$ such that $g_{\mid A}=f_{\mid A}$ and $h_{\mid \operatorname{dom}(f) \cap B}=f_{\mid \operatorname{dom}(f) \cap B}$. We have

$$
\begin{gathered}
p_{\operatorname{ran} f}=p_{f(A)}+p_{f(\operatorname{dom} f \cap B)} \text { and } \\
U_{f}=p_{f(A)} U_{f_{\mid A}}+p_{f(\operatorname{dom}(f) \cap B)} U_{f_{\operatorname{dom}(f) \cap B}}=p_{f(A)} U_{g}+p_{f(\operatorname{dom}(f) \cap B)} U_{h} .
\end{gathered}
$$

Thus

$$
U_{f}-p_{\operatorname{ran} f}=p_{f(A)}\left(U_{g}-1\right)+p_{f(\operatorname{dom} f \cap B)}\left(U_{h}-1\right) .
$$

Proposition 6.3. Let $M$ be a $\Gamma$-module. Then

$$
H_{0}(\Gamma / \mathcal{P}, M)=M_{\mathcal{E}}=M_{\mathcal{E}^{*}} .
$$

Proof. Immediate from Lemma 6.2.

6.3. Hochschild homology. We recall the basic definitions for Hochschild homology of algebras over a noncommutative base ring ([17, §1.2.11]). If $N$ is a $B \otimes B^{o p}$ module, we write

$$
\begin{aligned}
{[b, x] } & =b x-x b \quad(b \in B, x \in N), \\
{[B, N] } & =\left\{\sum_{i=1}^{n}\left[b_{i}, x_{i}\right]: b_{i} \in B, x_{i} \in N, n \geq 1\right\}, \\
N_{B} & =N /[B, N] .
\end{aligned}
$$


Next let $A \rightarrow B$ be a unital ring homomorphism. Recall from [17, §1.2.11] that the Hochschild homology of $B$ relative to $A$ with coefficients in $N, H H_{*}(B / A, N)=$ $\pi_{*} C(B / A, M)$, is the homotopy of the simplicial $\mathbb{Z}$-module which is given in dimension $n$ by

$$
C_{n}(B / A, N)=\left(N \otimes_{A} B^{\otimes_{A} n}\right)_{A},
$$

with the following face and degeneracy maps

$$
\begin{gathered}
\mu_{i}: C_{n+1}(B / A, N) \rightarrow C_{n}(B / A, N), \\
\mu_{i}\left(x_{0} \otimes \cdots \otimes x_{n+1}\right)=\left\{\begin{array}{cc}
x_{0} \otimes \cdots \otimes x_{i} x_{i+1} \otimes \cdots \otimes x_{n+1} & i \leq n \\
x_{n+1} x_{0} \otimes \cdots \otimes x_{n} & i=n+1
\end{array}\right. \\
s_{i}: C_{n}(B / A, N) \rightarrow C_{n+1}(B / A, N), \quad(0 \leq i \leq n) \\
s_{i}\left(x_{0} \otimes \cdots \otimes x_{n}\right)=x_{0} \otimes \cdots \otimes x_{i} \otimes 1 \otimes x_{i+1} \otimes \cdots \otimes x_{n} .
\end{gathered}
$$

We write $b$ for the alternating sum of the face maps, and $H H(B / A, N)$ for the resulting chain complex. Thus

$$
H H_{*}(B / A, N)=H_{*}(H H(B / A, N))
$$

is the Hochschild homology of $B / A$ with coefficients $N$. If $A$ is commutative and $B$ is central as an $A$-bimodule, then $B \otimes_{A} B^{o p}$ is a ring. If furthermore, $B$ happens to be flat as a left $A$-module, then

$$
H H_{*}(B / A, N)=\operatorname{Tor}_{*}^{B \otimes_{A} B^{o p}}(B, N) .
$$

Note this is the case, for example, if $A$ is a field. We shall write $H H_{*}(B, N)$ for $H H_{*}(B / \mathbb{Z}, N)$.

Remark 6.4. If $A$ and $B$ are commutative and $M$ is a central bimodule, then $C(B / A, M)=M \otimes_{B} C(B / A, B)$.

Lemma 6.5. (cf. [17, Theorem 1.12.13]) Let $k$ be a field, $A \rightarrow B$ a homomorphism of unital $k$-algebras, and $N$ a $B \otimes_{k} B^{o p}$-module. Assume that $A$ is a filtering colimit of separable $k$-algebras. Then

$$
H H_{*}(B / k, N)=H H_{*}(B / A, N) .
$$

Proof. It suffices to show that $B \otimes_{A} B^{o p}$ is flat as a $B \otimes_{k} B^{o p}$-module. By hypothesis $A=\operatorname{colim}_{i} A_{i}$ is a filtering colimit of separable algebras. Hence $B \otimes_{A} B^{o p}=$ $\operatorname{colim}_{i} B \otimes_{A_{i}} B^{o p}$, so it suffices to prove that if $k \subset A$ is separable then $B \otimes_{A} B$ is flat over $B \otimes_{k} B^{o p}$, and this is well known.

Example 6.6. If $k$ is a field, $A$ is a unital $\mathcal{P}(k)$-algebra, and $N$ is an $A \otimes_{k}$ $A^{o p}$-module, then $H H_{*}(A / k, N)=H H_{*}(A / \mathcal{P}(k), N)$, by Proposition 4.1 and Lemma 6.5. If $A \supset \mathbb{Q}$, then $H H_{*}(A, N)=H H_{*}(A / \mathbb{Q}, N)$ and $H H_{*}(A / \mathcal{P}, N)=$ $H H_{*}(A / \mathcal{P}(\mathbb{Q}), N)$, whence we also have $H H_{*}(A, N)=H H_{*}(A / \mathcal{P}, N)$. 
6.4. Hochschild homology of crossed products with $\Gamma$. In this subsection $k$ is a field and, as in (2.2.7), $R$ is an Emb-bundle over $k$; that is, $R$ is a $k$-algebra with a $k$-linear action of Emb so that $R$ is an Emb-bundle. We also fix an $R$-bimodule $M$, central as a $\mathcal{P}$-bimodule, together with a left action of Emb

$$
\mathrm{Emb} \times M \rightarrow M, \quad(f, m) \mapsto f_{*}(m) .
$$

We require that this action induce a $\Gamma$-module structure on $M$ which is covariant in the sense that

$$
f_{*}(r m s)=f_{*}(r) f_{*}(m) f_{*}(s) \quad(r, s \in R, m \in M) .
$$

In this situation, we can form the crossed product $M \#_{\mathcal{P}} \Gamma$; this is the $R \#_{\mathcal{P}} \Gamma$-bimodule consisting of $M \otimes_{\mathcal{P}} \Gamma$ equipped with the following left and right actions of $R \#_{\mathcal{P}} \Gamma$

$$
\left(a \# U_{f}\right)\left(m \# U_{g}\right)=a f_{*}(m) \# U_{f g}, \quad\left(m \# U_{g}\right)\left(a \# U_{f}\right)=m g_{*}(a) \# U_{g f} .
$$

Observe that, as $R$ is assumed to be a $k$-algebra, $M \#_{\mathcal{P}} \Gamma=M \#_{\mathcal{P}(k)} \Gamma(k)$. We are interested in the Hochschild homology of $R \#_{\mathcal{P}} \Gamma$ with coefficients in $M \#_{\mathcal{P}} \Gamma$, which by Example 6.6 is computed by the simplicial $\mathcal{P}(k)$-module $C\left(R \#_{\mathcal{P}} \Gamma / \mathcal{P}(k), M \#_{\mathcal{P}} \Gamma\right)$. On the other hand it is not hard to check, using (6.4.1) and the definition of Emb-bundle, that the diagonal action of Emb on $C(R / k)$ descends to an action of $\Gamma$ on $C(R / \mathcal{P}(k))$. Hence we may also consider the bisimplicial module $\perp(\Gamma / \mathcal{P}, C(R / \mathcal{P}(k), M))$ which results from applying the functor $\perp(\Gamma / \mathcal{P},-)$ dimension-wise to the simplicial module $C(R / \mathcal{P}(k), M)$. The diagonal of this bisimplicial module is

$$
\begin{aligned}
& \operatorname{diag}(\perp(\Gamma / \mathcal{P}, C(R / \mathcal{P}(k), M)))_{n} \\
& \quad=\perp^{n}\left(\Gamma / \mathcal{P}, C_{n}(R / \mathcal{P}(k), M)\right)=\left(M \otimes_{\mathcal{P}} R^{\otimes_{\mathcal{P}(k)} n}\right)_{\mathcal{P}} \otimes_{\mathcal{P}} \Gamma^{\otimes_{\mathcal{P}} n},
\end{aligned}
$$

with faces $\mu_{i} \partial_{i}$ and degeneracies $s_{i} \delta_{i}$. The simplicial module

$$
\operatorname{diag}(\perp(\Gamma / \mathcal{P}, C(R / \mathcal{P}(k), M)))
$$

is a model for the hyperhomology of $\Gamma / \mathcal{P}$ with $C(R / \mathcal{P}(k), M)$ coefficients. Hence, if $\mathbb{H}(\Gamma / \mathcal{P}, C(R / \mathcal{P}(k), M))$ is any other such model, we have a quasi-isomorphism

$$
\mathbb{H}(\Gamma / \mathcal{P}, C(R / \mathcal{P}(k), M)) \stackrel{\sim}{\longrightarrow} \operatorname{diag}(\perp(\Gamma / \mathcal{P}, C(R / \mathcal{P}(k), M)) .
$$

Observe that any element of $\operatorname{diag}(\perp(\Gamma / \mathcal{P}, C(R / \mathcal{P}(k), M)))_{n}$ can be written as a sum of congruence classes of elementary tensors of the form

$$
x=a_{0} \otimes a_{1} \otimes \cdots \otimes a_{n} \otimes f_{1} \otimes \cdots \otimes f_{n},
$$

where $a_{0} \in M, a_{i} \in R$, and $f_{i} \in \operatorname{Emb}(i \geq 1)$ are such that

$$
\begin{array}{cl}
\epsilon_{r}\left(f_{i}\right)=\epsilon_{l}\left(f_{i+1}\right) & (1 \leq i \leq n-1), \\
a_{j} \epsilon_{l}\left(f_{1}\right)=a_{j} & (0 \leq j \leq n) .
\end{array}
$$


Next we define a map

$$
\phi: \operatorname{diag}\left(\perp(\Gamma / \mathcal{P}, C(R / \mathcal{P}(k), M)) \rightarrow C\left(R \#_{\mathcal{P}} \Gamma / \mathcal{P}(k), M \#_{\mathcal{P}} \Gamma\right) .\right.
$$

For $x$ as in (6.4.2), we put

$$
\phi([x])=\left[a_{0} \# f_{1} \otimes f_{1}^{\dagger}\left(a_{1}\right) \# f_{2} \otimes \cdots \otimes\left(f_{1} \cdots f_{n}\right)^{\dagger}\left(a_{n}\right) \#\left(f_{1} \cdots f_{n}\right)^{\dagger}\right] .
$$

Here [] denotes congruence class.

Proposition 6.7. The assignment (6.4.3) gives a simplicial isomorphism

$$
\phi: \operatorname{diag}(\perp(\Gamma / \mathcal{P}, C(R / \mathcal{P}(k), M))) \stackrel{\cong}{\longrightarrow} C\left(R \#_{\mathcal{P}} \Gamma / \mathcal{P}(k), M \#_{\mathcal{P}} \Gamma\right) .
$$

In particular, we have a quasi-isomorphism

$$
\mathbb{H}(\Gamma / \mathcal{P}, H H(R / \mathcal{P}(k), M)) \stackrel{\sim}{\longrightarrow} H H\left(R \#_{\mathcal{P}} \Gamma / \mathcal{P}(k), M \#_{\mathcal{P}} \Gamma\right) .
$$

Proof. First of all, we must check that (6.4.3) gives a well-defined simplicial homomorphism. To do this, one checks first that formula (6.4.3) defines a simplicial homomorphism

$$
\hat{\phi}: \operatorname{diag}(\perp(\mathbb{Z}[\mathrm{Emb}], C(R, M))) \rightarrow C(R \# \mathrm{Emb}, M \# \mathrm{Emb}) .
$$

Then one observes that it passes down to the quotient, inducing a map $\phi: \operatorname{diag}(\perp$ $(\Gamma / \mathcal{P}, C(R / \mathcal{P}(k), M))) \rightarrow C\left(R \#_{\mathcal{P}} \Gamma / \mathcal{P}(k), M \#_{\mathcal{P}} \Gamma\right)$. Next note that the image of $\hat{\phi}$ is contained in the simplicial subgroup

$$
S \subset C(R \# \text { Emb, } M \# \text { Emb })
$$

given in dimension $n$ by

$$
S_{n}=\operatorname{span}\left\{\left[a_{0} \# f_{0} \otimes \cdots \otimes a_{n} \# f_{n}\right]: f_{i} \in \mathrm{Emb}, \quad a_{i} \in R, \quad f_{0} \cdots f_{n} \in 2^{\mathbb{N}}\right\} .
$$

To prove that $\phi$ is surjective, we must show that

$$
S \rightarrow C\left(R \#_{\mathcal{P}} \Gamma / \mathcal{P}(k), M \#_{\mathcal{P}} \Gamma\right)
$$

is surjective. Any element of $C\left(R \#_{\mathcal{P}} \Gamma / \mathcal{P}(k), M \#_{\mathcal{P}} \Gamma\right)$ can be written as a linear combination of classes of elementary tensors of the form

$$
y=a_{0} \# f_{0} \otimes \cdots \otimes a_{n} \# f_{n},
$$

such that the following conditions are satisfied for $0 \leq i \leq n-1$ and $0 \leq j \leq n$ :

$$
\epsilon_{r}\left(f_{i}\right)=\epsilon_{l}\left(f_{i+1}\right), \quad \epsilon_{r}\left(f_{n}\right)=\epsilon_{l}\left(f_{0}\right) \quad a_{j}=a_{j} \epsilon_{l}\left(f_{j}\right) .
$$

Let $f=f_{0} \cdots f_{n}$; then $\operatorname{dom}(f)=\operatorname{ran}(f)=\operatorname{ran}\left(f_{0}\right)=\operatorname{dom}\left(f_{n}\right)$. Let

$$
\mathbb{N} \supset A=\{x \in \operatorname{dom}(f): f(x)=x\} .
$$


If $A=\operatorname{dom}(f)$ then $f \in 2^{\mathbb{N}}$, and thus the element (6.4.4) belongs to $S$. Otherwise, by Zorn's Lemma, there exists $\emptyset \neq B \subset \operatorname{dom}(f)$ maximal with the property that $f(B) \cap B=\emptyset$. Clearly $A \cap B=\emptyset$; let $C=\operatorname{dom}(f) \backslash(A \sqcup B)$. Then $f(B) \subset C$, $f(C) \subset B$, and $p_{\operatorname{dom}(f)}=p_{A}+p_{B}+p_{C}$. Hence we have

$$
[y]=\left[p_{\operatorname{dom}(f)} y p_{\operatorname{dom}(f)}\right]=\left[p_{A} y p_{A}\right]=\left[a_{0} \# g_{0} \otimes \cdots \otimes a_{n} \# g_{n}\right],
$$

for $g_{n}=\left(f_{n}\right)_{\mid A}$ and $g_{i}=\left(f_{i}\right)_{\mid f_{i+1} \cdots f_{n}(A)}(0 \leq i \leq n-1)$. In particular $g_{0} \cdots g_{n}=p_{A}$. Thus $\phi$ is surjective. To prove it is injective, define a map

$$
\psi: C\left(R \#_{\mathcal{P}} \Gamma / \mathcal{P}(k), M \#_{\mathcal{P}} \Gamma\right) \rightarrow \operatorname{diag}(\perp(\Gamma / \mathcal{P}, C(R / \mathcal{P}(k), M)))
$$

as follows. For $y$ as in (6.4.4) satisfying the conditions (6.4.5) and such that $f_{0} \cdots f_{n} \in 2^{\mathbb{N}}$, put

$$
\psi([y])=\left[a_{0} \otimes f_{0}\left(a_{1}\right) \otimes \cdots \otimes\left(f_{0} \cdots f_{n-1}\right)\left(a_{n}\right) \otimes f_{0} \otimes \cdots \otimes f_{n-1}\right] .
$$

One checks that $\psi$ is well-defined and that $\psi \phi=i d$.

Corollary. Assume that $R$ is commutative and that $M$ is a central $R$-bimodule. Then

$$
H H_{0}\left(R \#_{\mathcal{P}} \Gamma, M \#_{\mathcal{P}} \Gamma\right)=M_{\mathcal{E}} .
$$

Proof. By Proposition 6.7,

$$
H H_{0}\left(R \#_{\mathcal{P}} \Gamma, M \#_{\mathcal{P}} \Gamma\right)=H_{0}\left(\Gamma / \mathcal{P}, H H_{0}(R, M)\right) .
$$

By our assumptions on $R$ and $M, H H_{0}(R, M)=M$. Finally we have $H_{0}(\Gamma / \mathcal{P}, M)=M_{\mathcal{E}}$, by Proposition 6.3.

6.5. Comparing the $0^{t h}$-homology of $\left(\Gamma^{\infty}, I_{S}\right)$ and that of $\left(\mathcal{B}: J_{S}\right)$.

Proposition 6.8. Let $S \triangleleft \ell^{\infty}$ be a symmetric ideal and let $J_{S} \triangleleft \mathcal{B}=\mathcal{B}\left(\ell^{2}\right)$ be the corresponding ideal of bounded operators in $\ell^{2}$. Then the inclusion $\Gamma^{\infty} \subset \mathcal{B}$ induces an isomorphism

$$
H H_{0}\left(\Gamma^{\infty}, I_{S}\right) \stackrel{\cong}{\longrightarrow} H H_{0}\left(\mathcal{B}, J_{S}\right) .
$$

Proof. By Proposition 2.3 Corollary 6.4, the inclusion diag : $S \rightarrow I_{S}$ descends to a bijection

$$
S_{\mathcal{E}} \stackrel{\cong}{\longrightarrow} H H_{0}\left(\Gamma^{\infty}, I_{S}\right) .
$$

By [13, Theorem 5.12] the composite of (6.5.1) with the map induced by the inclusion $I_{S} \subset J_{S}$ is an isomorphism.

Corollary. The map $H C_{0}\left(\Gamma^{\infty}: I_{S}\right) \rightarrow H C_{0}\left(\mathcal{B}: J_{S}\right)$ is an isomorphism. 
Proof. It follows from Proposition 6.8 and the fact that, if $R$ is a unital ring and $I \triangleleft R$ is an ideal then

$$
H H_{0}(R: I)=H C_{0}(R: I)=I /[R, I] .
$$

Lemma 6.9. Let $p>0$. Then:

$$
\begin{gathered}
H C_{0}\left(\Gamma^{\infty}: I_{\ell p+}\right)= \begin{cases}\mathbb{C} & p<1 \\
0 & p \geq 1\end{cases} \\
H C_{0}\left(\Gamma^{\infty}: I_{\ell} \ell^{p-}\right)= \begin{cases}\mathbb{C} & p \leq 1 \\
0 & p>1\end{cases} \\
H C_{0}\left(\Gamma^{\infty}: I_{\ell p}\right)=\left\{\begin{array}{cc}
\mathbb{C} & p<1 \\
\mathbb{C} \oplus \mathbb{V} & p=1 \\
0 & p>1 .
\end{array}\right.
\end{gathered}
$$

Here $\mathbb{V}$ is a $\mathbb{C}$-vector space of uncountable dimension.

Proof. It follows from Corollary 6.5 and [24, pp. 492-493].

6.6. Cyclic homology of $R \#_{\mathcal{P}} \Gamma$. Now we go back to the general situation of Subsection 6.4. So $k$ is a field and $R$ is an Emb-bundle over $k$. Let $M$ be a right $\Gamma$-module. Consider the simplicial module $\perp(\Gamma / \mathcal{P}, M)$. Every element of $\perp_{n}(\Gamma / \mathcal{P}, M)$ can be written as a sum of elementary tensors

$$
x=m \otimes f_{1} \otimes \cdots \otimes f_{n}
$$

with $m \in M, f_{i} \in \mathrm{Emb}$, and $\operatorname{dom}\left(f_{i}\right)=\operatorname{ran}\left(f_{i+1}\right)(i<n)$. For $x$ as above, put

$$
\tau_{n}(x)=(-1)^{n} m\left(f_{1} \cdots f_{n}\right) \otimes\left(f_{1} \cdots f_{n}\right)^{\dagger} \otimes f_{1} \otimes \cdots \otimes f_{n-1} .
$$

One checks that the assignment (6.6.1) gives a well-defined endomorphism of $\perp_{n}(\Gamma / \mathcal{P}, M)$, and that the cyclic identities [17, 2.5.1.1] hold. Thus the simplicial ( $k$-)module $\perp(\Gamma / \mathcal{P}, M)$, equipped with the cyclic operators $\tau_{n}(n \geq 0)$, is a cyclic module. In general if $\mathcal{C}$ is any cyclic module, then we can equip $\mathcal{C}$ with a map $B: \mathcal{C} \rightarrow \mathcal{C}[+1]$ called the Connes' operator, which, together with the usual boundary $b: \mathcal{C} \rightarrow \mathcal{C}[-1]$ given by the alternating sum of the face maps, satisfy $b^{2}=B^{2}=[b, B]=0$. When $\mathcal{C}=\perp(\Gamma / \mathcal{P}, M)$, we write $\partial$ and $\mathcal{B}$ for the operators $b$ and $B$. The Hochschild complex of a cyclic module $\mathcal{C}$ is $H H(\mathcal{C})=(\mathcal{C}, b)$. The cyclic and negative cyclic complexes are the complexes given in dimension $n$ by $H C(\mathcal{C})_{n}=\bigoplus_{m \geq 0} \mathcal{C}_{n-2 m}$ and $H N(\mathcal{C})_{n}=\prod_{m \geq 0} \mathcal{C}_{n+2 m}$; 
they are equipped with the boundary $b+B$. Observe that $H C(\mathcal{C})$ is also equipped with a chain map $S: H C(\mathcal{C}) \rightarrow H C(\mathcal{C})[-2]$ defined by the obvious projections $H C(\mathcal{C})_{n} \rightarrow H C(\mathcal{C})_{n-2}$. If $C$ is another chain complex equipped with a chain map $S: C \rightarrow C[-2]$, then by a map of $S$-complexes $C \rightarrow H C(\mathcal{C})$ we understand a chain map which commutes with $S$.

Proposition 6.10. There is a natural quasi-isomorphism of $S$-complexes $(H C(\perp$ $(\Gamma / \mathcal{P}, M)), \partial) \rightarrow(H C(\perp(\Gamma / \mathcal{P}, M)), \partial+\mathcal{B})$.

Proof. View $\mathcal{C}=\perp(\Gamma / \mathcal{P}, M)$ as a cyclic module. Consider the projection

$$
\pi: H N(\mathcal{C})_{n}=\prod_{m \geq 0} \mathcal{C}_{n+2 m} \rightarrow \mathcal{C}_{n}=H H(\mathcal{C})_{n}
$$

Observe that $\pi(b+\mathcal{B})=b \pi$. Proceed as in [11, §3.1] to define a chain map $\Upsilon: H H(\mathcal{C}) \rightarrow H N(\mathcal{C})$ such that $\pi \Upsilon=1$. We have a chain map $\theta^{n}: H N(\mathcal{C}) \rightarrow$ $H C(\mathcal{C})[2 n](n \geq 0)$ given by the composite

$$
\begin{aligned}
\theta^{n}: H N(\mathcal{C})_{p}=\prod_{m \geq 0} \mathcal{C}_{p+2 m} \rightarrow \bigoplus_{m=0}^{n} \mathcal{C}_{p+2 m} & \\
& \subset \bigoplus_{q \geq 0} \mathcal{C}_{p+2(n-q)}=H C(\mathcal{C})_{p+2 n}
\end{aligned}
$$

The map of the proposition is

$$
\sum_{n=0}^{\infty} \theta^{n} \Upsilon:(H C(\mathcal{C}), \partial)=\bigoplus_{n \geq 0} H H(\mathcal{C})[-2 n] \rightarrow(H C(\mathcal{C}), b+\mathcal{B})
$$

Theorem 6.11. Let $k$ be a field and $R$ an Emb-bundle over $k$. There is a natural zig-zag of quasi-isomorphisms

$$
\mathbb{H}(\Gamma / \mathcal{P}, H C(R / \mathcal{P}(k))) \stackrel{\sim}{\longrightarrow} H C\left(R \#_{\mathcal{P}} \Gamma / k\right) .
$$

Proof. Consider the bicyclic module

$$
\mathcal{C}_{*, *}:([m],[n]) \mapsto \perp_{m}\left(\Gamma / \mathcal{P}, C_{n}(R / \mathcal{P}(k))\right) .
$$

It follows from Proposition 6.10 that the total cyclic complex

$$
T=\left(H C\left(\mathcal{C}_{*, *}\right), b+\partial+B+\mathcal{B}\right)
$$

is quasi-isomorphic to

$$
\left(H C\left(\mathcal{C}_{*, *}\right), b+\partial+B\right)
$$


which in turn is a model for $\mathbb{H}(\Gamma / \mathcal{P}, H C(R / \mathcal{P}(k)))$. By the cylindrical version of the Eilenberg-Zilber theorem ([16, Theorem 3.1]), the complex $T$ is $S$-equivalent to the $H C$-complex of the diagonal $\Delta$ of (6.6.2). By Proposition (6.7), the map (6.4.3) is an isomorphism of simplicial modules $\Delta \cong C\left(R \#_{\mathcal{P}} \Gamma / \mathcal{P}(k)\right)$; one checks that it is actually an isomorphism of cyclic modules. Finally, by Example 6.6, the projection $C\left(R \#_{\mathcal{P}} \Gamma / k\right) \rightarrow C\left(R \#_{\mathcal{P}} \Gamma / \mathcal{P}(k)\right)$ induces a quasi-isomorphism

$$
H C\left(R \#_{\mathcal{P}} \Gamma / k\right) \rightarrow H C\left(R \#_{\mathcal{P}} \Gamma / \mathcal{P}(k)\right) .
$$

Corollary. Let $\mathfrak{A}$ be a bornological algebra and $S \triangleleft \ell^{\infty}$ a symmetric ideal. Then

$$
H C_{*}\left(\Gamma^{\infty}(\mathfrak{A}): I_{S(\mathfrak{A})}\right)=\mathbb{H}_{*}\left(\Gamma / \mathcal{P}: H C\left(\left(\ell^{\infty}(\mathfrak{A}): S(\mathfrak{A})\right) / \mathcal{P}\right)\right) .
$$

Proof. By Proposition 2.3, we have $\Gamma^{\infty}(\mathfrak{A})=\ell^{\infty}(\mathfrak{A}) \#_{\mathcal{P}} \Gamma$ and $I_{S(\mathfrak{A})}=S(\mathfrak{A}) \#_{\mathcal{P}} \Gamma$. Now apply Theorem 6.11 and take fibers.

6.7. Hodge decomposition. If $R$ is a commutative $\mathbb{Q}$-algebra, then there are defined Adams operations on $C(R)$, and we have an eigenspace decomposition [17, Theorems 4.5.10 and 4.6.7]

$$
C(R)=\bigoplus_{p \geq 0} C^{(p)}(R)
$$

called the Hodge decomposition. We have $C_{n}^{(p)}=0$ for $n<p$ and each $C^{(p)}$ is a graded $R$-submodule, closed under the Hochschild boundary map $b$. Thus, if $M$ is a central $R$-bimodule, for $H H^{(p)}(R, M)=M \otimes_{R}\left(C^{(p)}(R), b\right)$ we have

$$
H H_{n}(R, M)=\bigoplus_{p \geq 0}^{n} H H_{n}^{(p)}(R, M) .
$$

The Connes operator $B$ sends $C^{(p)}$ to $C^{(p+1)}$. Thus, we have a direct sum decomposition of the cyclic complex

$$
H C(R)=\bigoplus_{p=0}^{\infty} H C^{(p)}(R)
$$

where

$$
H C^{(p)}(R)_{n}=\bigoplus_{p \geq 0}^{n} C_{n-2 p}^{(n-p)}(R) .
$$


Hence for $H C_{*}^{(p)}(R)=H_{*}\left(H C^{(p)}(R)\right)$,

$$
H C_{n}(R)=\bigoplus_{p=0}^{n} H C_{n}^{(p)}(R) .
$$

Let $\left(\Omega_{R}^{*}, d\right)$ be the $D G A$ of (absolute) Kähler differential forms. There is a natural map of mixed complexes

$$
\begin{gathered}
\mu:(C(R), b, B) \rightarrow\left(\Omega_{R}, 0, d\right) \\
\mu\left(x_{0} \otimes \cdots \otimes x_{n}\right)=(1 / n !) x_{0} d x_{1} \wedge \cdots \wedge d x_{n} .
\end{gathered}
$$

Let $M$ be a central $R$-bimodule; the map $\mu$ induces isomorphisms

$$
\begin{gathered}
H H_{n}^{(n)}(R, M)=M \otimes_{R} \Omega_{R}^{n} \\
\text { and } H C_{n}^{(n)}(R)=\Omega_{R}^{n} / d\left(\Omega_{R}^{n-1}\right) .
\end{gathered}
$$

We say that $R$ is homologically smooth if (6.7.2) is a quasi-isomorphism.

Remark 6.12. If $R$ happens to also be an algebra over $\mathcal{P}$, then the Hodge decomposition above induces a similar decomposition on $H H(R / \mathcal{P}, M)$ and $H C(R / \mathcal{P})$, so that $H H^{(p)}(R, M) \rightarrow H H^{(p)}(R / \mathcal{P}, M)$ and $H H^{(p)}(R, M) \rightarrow$ $H H^{(p)}(R / \mathcal{P})$ are quasi-isomorphisms. Moreover $\Omega_{R} \rightarrow \Omega_{R / \mathcal{P}}$ is an isomorphism.

Example 6.13. Let $R$ be a unital commutative complex $C^{*}$-algebra over $\mathbb{C}$. It was proved in [10, Thm. 8.2.6] that $R$, regarded as a $\mathbb{Q}$-algebra, is homologically smooth. In particular this applies when $R=\ell^{\infty}$. Moreover, by [10, proof of Prop. 5.2.2], $\ell^{\infty}$ is a filtering colimit of smooth $\mathbb{C}$-algebras. It follows that $\Omega_{\ell^{\infty}}^{n}$ is a flat $\ell^{\infty}$-module for every $n$. Hence

$$
H H_{n}\left(\ell^{\infty}, M\right)=M \otimes_{\ell \infty} \Omega_{\ell \infty}^{n}
$$

for every central bimodule $M$.

Now assume that the commutative $\mathbb{Q}$-algebra $R$ is an Emb-bundle. Then by Proposition 6.7, Theorem 6.11, and naturality of the Hodge decomposition, we have quasi-isomorphisms

$$
\begin{aligned}
H H\left(R \#_{\mathcal{P}} \Gamma, M \#_{\mathcal{P}} \Gamma\right) \stackrel{\sim}{\longrightarrow} \bigoplus_{p \geq 0} \mathbb{H}\left(\Gamma / \mathcal{P}, H H^{(p)}(R / \mathcal{P}, M)\right) \\
\text { and } H C\left(R \#_{\mathcal{P}} \Gamma\right) \stackrel{\sim}{\longrightarrow} \bigoplus_{p \geq 0} \mathbb{H}\left(\Gamma / \mathcal{P}, H C^{(p)}(R / \mathcal{P})\right) .
\end{aligned}
$$

Put

$$
\begin{gathered}
H H_{n}^{(p)}\left(R \#_{\mathcal{P}} \Gamma, M \#_{\mathcal{P}} \Gamma\right)=\mathbb{H}_{n}\left(\Gamma / \mathcal{P}, H H^{(p)}(R / \mathcal{P}, M)\right), \\
H C_{n}^{(p)}\left(R \#_{\mathcal{P}} \Gamma\right)=\mathbb{H}_{n}\left(\Gamma / \mathcal{P}, H C^{(p)}(R / \mathcal{P})\right) .
\end{gathered}
$$


We have decompositions

$$
\begin{aligned}
H H_{n}\left(R \#_{\mathcal{P}} \Gamma, M \#_{\mathcal{P}} \Gamma\right) & =\bigoplus_{p=0}^{n} H H_{n}^{(p)}\left(R \#_{\mathcal{P}} \Gamma, M \#_{\mathcal{P}} \Gamma\right), \\
H C_{n}\left(R \#_{\mathcal{P}} \Gamma\right) & =\bigoplus_{p=0}^{n} H C_{n}^{(p)}\left(R \#_{\mathcal{P}} \Gamma\right) .
\end{aligned}
$$

If follows from (6.7.3), (6.7.4), and Proposition 6.3 that

$$
\begin{gathered}
H H_{n}^{(n)}\left(R \#_{\mathcal{P}} \Gamma, M \#_{\mathcal{P}} \Gamma\right)=\left(M \otimes_{R} \Omega_{R}^{n}\right)_{\mathcal{E}}, \\
H C_{n}^{(n)}\left(R \#_{\mathcal{P}} \Gamma\right)=\left(\Omega_{R}^{n} / d \Omega_{R}^{n-1}\right)_{\mathcal{E}} .
\end{gathered}
$$

\section{The relative cyclic homology $H C_{*}\left(\Gamma^{\infty}(\mathfrak{A}): I_{S(\mathfrak{A})}\right)$}

7.1. The Quillen spectral sequence. Let $R$ be a unital $\mathbb{Q}$-algebra and $I \triangleleft R$ a two-sided ideal, flat both as a right and as a left ideal. Then

$$
I^{\otimes_{R}^{n}} \cong I^{n} .
$$

Using the isomorphism above and flatness again we see that if $P \stackrel{\sim}{\longrightarrow} I$ is a projective bimodule resolution, then $Q=P^{\otimes_{R}^{n}} \stackrel{\sim}{\longrightarrow} I^{n}$ is again a resolution. Hence modding out $Q$ by the commutator subspace $[Q, R]$ we obtain a complex which computes $H H_{*}\left(R, I^{n}\right)$ and which has a natural action of $\mathbb{Z} / n \mathbb{Z}$ via permutation of factors. Following Quillen [19, pp. 210] we shall write $H H_{*}\left(R, I^{n}\right)_{\sigma}$ for the coinvariants of this action. Quillen introduced a first quadrant spectral sequence (see [19, Proposition 2.16 and Theorem 4.3]),

$$
E_{p, q}^{1}=\left\{\begin{array}{cc}
H C_{q}(R) & p=0 \\
H H_{q-p+1}\left(R, I^{p}\right)_{\sigma} & p \geq 1,
\end{array}\right.
$$

which converges to $H C_{p+q}(R / I)$. For example, every ideal $J \triangleleft \mathcal{B}=\mathcal{B}\left(\ell^{2}\right)$ of the algebra of bounded operators is flat; $M$. Wodzicki has used this spectral sequence, together with the results of [13], to study the relative cyclic homology groups $H C_{*}(\mathcal{B}: J)$. By Proposition 3.3, every ideal of $\Gamma^{\infty}$ is flat; by Proposition 3.5 and Examples 3.2, the same is true of $I_{c_{0}(\mathfrak{A})}$ and $I_{\ell^{\infty-(\mathfrak{A})}}$ for every unital Banach algebra $\mathfrak{A}$. In this subsection we shall use Quillen's spectral sequence to study the cyclic homology groups $H C_{*}\left(\Gamma^{\infty}: I_{S}\right)$. Proposition 7.1 below will play a role akin to that played by [24, Theorem 8] in the context of operator ideals. Let $\mathfrak{A}$ and $\mathfrak{B}$ be Banach algebras, and let $\hat{\otimes}$ be the projective tensor product. We have maps

$$
\begin{gathered}
\Gamma \otimes \Gamma \rightarrow \Gamma(\mathbb{N} \times \mathbb{N}), \quad U_{f} \otimes U_{g} \mapsto U_{f \times g}, \\
\otimes: \ell^{\infty}(\mathfrak{A}) \otimes \ell^{\infty}(\mathfrak{B}) \rightarrow \ell^{\infty}(\mathbb{N} \times \mathbb{N}, \mathfrak{A} \hat{\otimes} \mathfrak{B}), \quad(\alpha \otimes \beta)_{m, n}=\alpha_{n} \hat{\otimes} \beta_{m} .
\end{gathered}
$$


These two maps together induce

$$
\begin{aligned}
& \Gamma^{\infty}(\mathfrak{A}) \otimes \Gamma^{\infty}(\mathfrak{B}) \rightarrow \\
& \qquad \Gamma^{\infty}(\mathbb{N} \times \mathbb{N}, \mathfrak{A} \hat{\otimes} \mathfrak{B}):=\ell^{\infty}(\mathbb{N} \times \mathbb{N}, \mathfrak{A} \hat{\otimes} \mathfrak{B}) \#_{\mathcal{P}(\mathbb{N} \times \mathbb{N})} \Gamma(\mathbb{N} \times \mathbb{N}) .
\end{aligned}
$$

We write $\Gamma^{\infty}(\mathbb{N} \times \mathbb{N})=\Gamma^{\infty}(\mathbb{N} \times \mathbb{N}, \mathbb{C})$. In particular we have a map

$$
\Gamma^{\infty} \otimes \Gamma^{\infty} \rightarrow \Gamma^{\infty}(\mathbb{N} \times \mathbb{N}) .
$$

Proposition 7.1. (cf. [24, Theorem 8]) Let $S, T \triangleleft \ell^{\infty}$ be symmetric ideals, and let $\mathfrak{B}$ be a unital Banach algebra. Assume that

(i) The map (7.1.3) sends $S \otimes T \rightarrow T(\mathbb{N} \times \mathbb{N})$.

(ii) $S_{\mathcal{E}}=0$.

Then

$$
H H_{*}\left(\Gamma^{\infty}(\mathfrak{B}), I_{T(\mathfrak{B})}\right)=0 .
$$

Proof. Proceeding as in the proof of [1, Proposition 7.3.4], we obtain a commutative diagram

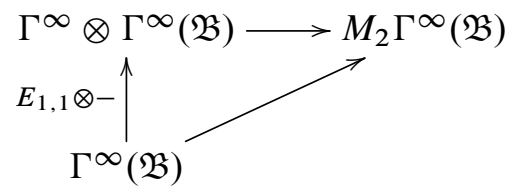

By hypothesis (i) this restricts to a commutative diagram

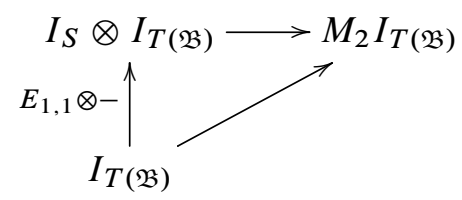

Now use hypothesis (ii), Morita invariance and the Künneth formula for Hochschild homology ([?Theorem 1.2.4]lod and [22, Proposition 9.4.1]), and induction, to conclude that $H H_{*}\left(\Gamma^{\infty}(\mathfrak{A}), I_{T(\mathfrak{A})}\right)=0$.

We shall need the following result of Dykema, Figiel, Weiss and Wodzicki, which follows by combining [13, Theorem 5.11(ii) and Theorem 5.12].

Proposition 7.2. ([13]) Let $S \triangleleft \ell^{\infty}$ be a symmetric ideal and let $\omega=(1 / n)_{n \geq 1}$ be the harmonic sequence. Then

$$
S_{\mathcal{E}}=0 \Longleftrightarrow \omega \otimes S \subset S(\mathbb{N} \times \mathbb{N}) .
$$




\section{Proposition 7.3.}

(i) $H C_{*}\left(\Gamma^{\infty}: I_{c_{0}}\right)=H C_{*}\left(\mathcal{B}: J_{c_{0}}\right)=0$.

(ii) $H C_{*}\left(\Gamma^{\infty}: I_{\ell}{ }^{\infty-}\right)=H C_{*}\left(\mathcal{B}: J_{\ell}{ }^{\infty-}\right)=0$.

(iii) Let $0<p<\infty, S \in\left\{\ell^{p}, \ell^{p-}, \ell^{p+}\right\}$,

$$
\begin{gathered}
m=\min \left\{n: H C_{n}\left(\Gamma^{\infty}: I_{S}\right) \neq 0\right\}, \\
\text { and } m^{\prime}=\min \left\{n: H C_{n}\left(\mathcal{B}: J_{S}\right) \neq 0\right\}
\end{gathered}
$$

Then $m=m^{\prime}$ and the map $H C_{m}\left(\Gamma^{\infty}: I_{S}\right) \rightarrow H C_{m}\left(\mathcal{B}: J_{S}\right)$ is an isomorphism.

Proof. Consider the spectral sequence (7.1.1) in the cases $R=\Gamma^{\infty}, \mathcal{B}$ and $I=I_{S}, J_{S}$ for each of the symmetric ideals $S$ of the proposition. We have $E_{0, *}^{1}=0$ since both $\Gamma^{\infty}$ and $\mathcal{B}$ are rings with infinite sums [1, §5]. In both (i) and (ii), we have $S^{2}=S$ and $\omega \otimes S \subset S(\mathbb{N} \times \mathbb{N})$ whence $E_{*, *}^{1}=0$, by Propositions 7.2 and 7.1 and [24, Theorem 8]. This gives (i) and (ii). In each of the cases considered in part (iii), we have $S \otimes S \subset S(\mathbb{N} \times \mathbb{N})$. Since $\omega \in \ell^{p}$ if and only if $p>1$ and since $\left(\ell^{p}\right)^{n}=\ell^{p / n}$, we have $H H_{*}\left(\Gamma^{\infty}, I_{\left(\ell^{p}\right)^{n}}\right)=H H_{*}\left(\mathcal{B},\left(\mathcal{L}^{p}\right)^{n}\right)=0$ for $p / n>1$, again by Propositions 7.2 and 7.1 and [24, Theorem 8]. The case $S=\ell^{p}$ follows from this and from Corollary 6.8. The remaining cases follow similarly.

Remark 7.4. Proposition 7.12 below provides a more detailed computation of $H C_{n}\left(\Gamma^{\infty}: I_{S}\right)$ for $S$ as in case iii) of Proposition 7.3 above.

Theorem 7.5. The comparison map $K_{*}\left(I_{S(\mathfrak{A})}\right) \rightarrow K H_{*}\left(I_{S(\mathfrak{A})}\right)$ is an isomorphism in the following cases:

(i) $S=c_{0}$ and $\mathfrak{A}$ is a $C^{*}$-algebra.

(ii) $S=\ell^{\infty-}$ and $\mathfrak{A}$ is a unital Banach algebra.

Proof. By Proposition 5.1 and Examples 5.2 and 5.3, $I_{S(\mathfrak{A})}$ is $H$-unital in both cases. Hence by (1.2) it suffices to show that $H C_{*}\left(\Gamma^{\infty}(\mathfrak{A}): I_{S(\mathfrak{A})}\right)=0$. As explained in the proof of Proposition 7.3, Proposition 7.2 implies that $S_{\mathcal{E}}=0$. Hence if $\mathfrak{A}$ is unital we are done by Propositions 3.5 and 7.1; in particular, part (ii) is proved. The nonunital case of (i) follows from the unital case using excision.

7.2. Computing $H C^{(p)}\left(\Gamma^{\infty}: I_{S}\right)$ in terms of differential forms. Let $S \triangleleft \ell^{\infty}$ be an ideal. Consider the subcomplex

$$
\begin{gathered}
\mathcal{F}_{p}(S) \subset \Omega_{\ell \infty} \\
\left(\mathcal{F}_{p}(S)\right)^{q}=\left\{\begin{array}{cc}
S^{p-q+1} \Omega_{\ell \infty}^{q} & p \geq q \\
\Omega_{\ell \infty}^{q} & q>p .
\end{array}\right.
\end{gathered}
$$


Write

$$
\begin{gathered}
D^{(p)}(S)_{q}=\left(\Omega_{\ell^{\infty}}^{-q} /\left(\mathcal{F}_{p}^{-q}(S)\right)\right. \\
L^{(p)}(S)_{q}=\mathcal{F}_{p-1}^{-q}(S) / \mathcal{F}_{p}^{-q}(S) .
\end{gathered}
$$

Note $L^{(p)}(S)$ and $D^{(p)}(S)$ are nonpositive chain complexes.

Theorem 7.6. Let $S \triangleleft \ell^{\infty}$ be a symmetric ideal. Then there are Emb-equivariant quasi-isomorphisms

$$
\begin{aligned}
H H^{(p)}\left(\ell^{\infty} / S\right) \stackrel{\sim}{\longrightarrow} L^{(p)}(S)[p] \\
H C^{(p)}\left(\ell^{\infty} / S\right) \stackrel{\sim}{\longrightarrow} D^{(p)}(S)[p] .
\end{aligned}
$$

Proof. Consider the skew-commutative graded algebra $\Lambda=\ell^{\infty} \oplus S$ with grading $\Lambda_{0}=\ell^{\infty}, \Lambda_{1}=S$. The inclusion $S \subset \ell^{\infty}$ defines a homogeneous $\ell^{\infty}$-linear derivation $\partial: \Lambda \rightarrow \Lambda[-1]$. Thus $\Lambda$ is a chain $D G A$, and the projection $\ell^{\infty} \rightarrow$ $\ell^{\infty} / S$ defines a quasi-isomorphism of cyclic modules $C(\Lambda, \partial) \stackrel{\sim}{\longrightarrow} C\left(\ell^{\infty} / S\right)$. By [7, Thms. 2.6 and 3.3] and Proposition 3.1, there are quasi-isomorphisms $C(\Lambda, \partial) \stackrel{\sim}{\longrightarrow}$ $\bigoplus_{p} L^{(p)}(S)[p]$ and $\mathfrak{B}(\Lambda, \partial) \stackrel{\sim}{\longrightarrow} \bigoplus_{p} D^{(p)}(S)[p]$; by [21] they are compatible with the Hodge decomposition. Finally, all these quasi-isomorphisms are natural, and thus Emb-equivariant.

\section{Theorem 7.7.}

$$
\begin{aligned}
& H C_{*}^{(p)}\left(\Gamma^{\infty}: I_{S}\right)=\mathbb{H}_{*+p}\left(\Gamma / \mathcal{P}, \mathcal{F}_{(p)}(S)\right) \\
& H H_{*}^{(p)}\left(\Gamma^{\infty}: I_{S}\right)=\mathbb{H}_{*+p+1}\left(\Gamma / \mathcal{P}, L_{(p)}(S)\right)
\end{aligned}
$$

Proof. It follows from (6.7.7) using Theorem 7.6 and the fact that $\Gamma^{\infty}$ is an infinite sum ring $([1, \S 5])$.

Corollary 7.8. There is a first quadrant homological spectral sequence

$$
{ }_{p} E_{m, n}^{1}=H_{n}\left(\Gamma / \mathcal{P}, S^{m+1} \Omega_{\ell \infty}^{p-m}\right) \Rightarrow H C_{m+n+p}^{(p)}\left(\Gamma^{\infty}: I_{S}\right) .
$$

Proof. This is the spectral sequence associated to $\mathbb{H}\left(\Gamma / \mathcal{P}, \mathcal{F}_{(p)}(S)\right)$. It is located in the first quadrant because as $\Gamma^{\infty}$ is an infinite sum ring,

$$
H H_{*}^{(q)}\left(\Gamma^{\infty}\right)=H_{*+q}\left(\Gamma / \mathcal{P}, \Omega_{\ell \infty}^{q}\right)=0
$$

\section{Corollary 7.9.}

$$
H C_{n}^{(n)}\left(\Gamma^{\infty}: I_{S}\right)=\left(S \Omega_{\ell^{\infty}}^{n} / d\left(S^{2} \Omega_{\ell^{\infty}}^{n-1}\right)\right)_{\mathcal{E}} .
$$

Proof. It follows from inspection of the second term of the spectral sequence of Corollary 7.8 , by using the fact that $H_{0}(\Gamma / \mathcal{P},-)=()_{\mathcal{E}}$ is right exact. 
7.3. The cases $S=\ell^{p}, \ell^{p \pm}$.

Lemma 7.10. Let $S \triangleleft \ell^{\infty}$ be a symmetric ideal. Then the map

$$
C\left(\Gamma / \mathcal{P}, S \Omega_{\ell^{\infty}}^{p}\right) \rightarrow C\left(\Gamma(\mathbb{N} \sqcup \mathbb{N}) / \mathcal{P}(\mathbb{N} \sqcup \mathbb{N}), S(\mathbb{N} \sqcup \mathbb{N}) \Omega_{\ell^{\infty}(\mathbb{N} \cup \mathbb{N})}^{p}\right)
$$

induced by the inclusion $\mathbb{N} \subset \mathbb{N} \sqcup \mathbb{N}$ into the first copy, is a quasi-isomor-phism.

Proof. Recall from Corollary 3 that every ideal of $\ell^{\infty}$ is flat, and from Example 6.13 that $\Omega_{\ell \infty}^{p}$ is a flat $\ell^{\infty}$-module. It follows that the map $S \otimes_{\ell \infty} \Omega_{\ell^{\infty}}^{p} \rightarrow S \Omega_{\ell \infty}^{p}$ is an isomorphism for every ideal $S$. Now the proof is immediate from [1, Lemma 7.3.1] and Lemma 6.1.

Lemma 7.11. Let $0 \neq S_{1}, S_{2} \subset \ell^{\infty}$ be symmetric ideals. Assume that $\left(S_{1}\right)_{\mathcal{E}}=0$ and that the map $\ell^{\infty} \otimes \ell^{\infty} \rightarrow \ell^{\infty}(\mathbb{N} \times \mathbb{N})$ sends $S_{1} \otimes S_{2} \rightarrow S_{2}(\mathbb{N} \times \mathbb{N})$. Then $H_{*}\left(\Gamma / \mathcal{P}, S_{2} \Omega_{\ell \infty}^{p}\right)=0(p \geq 0)$.

Proof. The proof follows using Lemma 7.10 and the argument of the proof of Proposition 7.1.

Let $p \in \mathbb{R}$; the following notation is used in the proposition below.

$$
[p]=\max \{n \in \mathbb{Z}: n \leq p\},\lfloor p\rfloor=\left\{\begin{array}{cc}
p-1 & p \in \mathbb{Z} \\
{[p]} & p \notin \mathbb{Z} .
\end{array}\right.
$$

\section{Proposition 7.12.}

(i) Let $p>0$ and let $S_{p}$ be either $\ell^{p}$ or $\ell^{p-}$. Then

$$
\begin{aligned}
& H C_{n}^{(q)}\left(\Gamma^{\infty}: I_{S_{p}}\right)= \\
& \qquad \begin{array}{cc}
0 & n<q+\lfloor p\rfloor \\
\left(S_{(p /(\lfloor p\rfloor+1))} \Omega_{\ell \infty}^{q-\lfloor p\rfloor} / d\left(S_{(p /(\lfloor p\rfloor+2))} \Omega_{\ell}^{q-\lfloor p\rfloor-1}\right)\right)_{\mathcal{E}} & n=q+\lfloor p\rfloor .
\end{array}
\end{aligned}
$$

In particular, the first nonzero group is

$$
H C_{2\lfloor p\rfloor}\left(\Gamma^{\infty}: I_{S_{p}}\right)=H C_{2\lfloor p\rfloor}^{\lfloor p\rfloor}\left(\Gamma^{\infty}: I_{S_{p}}\right)=H C_{0}\left(\Gamma^{\infty}: I_{S_{p /(\lfloor p\rfloor+1)}}\right)
$$

which was computed in 6.9.

(ii)

$$
\begin{aligned}
& H C_{n}^{(q)}\left(\Gamma^{\infty}: I_{\ell p+}\right)= \\
& \left\{\begin{array}{cc}
0 & n<q+[p] \\
\left(\ell^{(p /([p]+1))+} \Omega_{\ell \infty}^{q-[p]} / d\left(\ell^{(p /([p]+2))+} \Omega_{\ell \infty}^{q-[p]-1}\right)\right)_{\mathcal{E}} & n=q+[p] .
\end{array}\right.
\end{aligned}
$$

In particular, the first nonzero group is

$$
\begin{aligned}
H C_{2[p]}\left(\Gamma^{\infty}: I_{\ell^{p+}}\right)=H C_{2[p]}^{([p])}\left(\Gamma^{\infty}:\right. & \left.I_{\ell^{p+}}\right) \\
& =H C_{0}\left(\Gamma^{\infty}: I_{\ell^{(p /([p]+1))+}}\right)=\mathbb{C}
\end{aligned}
$$


Proof. This is a straightforward application of the spectral sequence of Corollary 7.8 together with Lemma 7.11 and Proposition 7.2.

\section{References}

[1] Beatriz Abadie and Guillermo Cortiñas, Homotopy invariance through small stabilizations, arXiv:1212.5901, 2013.

[2] Alcides Buss and Ruy Exel, Fell bundles over inverse semigroups and twisted étale groupoids, J. Operator Theory 67 (2012), no. 1, 153-205, Zbl 1249.46053 MR 2881538.

[3] J. W. Calkin, Two-sided ideals and congruences in the ring of bounded operators in Hilbert space, Ann. of Math. (2) 42 (1941), 839-873, Zbl 0063.00692 MR 5790.

[4] Henri Cartan and Samuel Eilenberg, Homological algebra, Princeton University Press, Princeton, N. J., 1956, Zbl 0075.24305 MR 77480.

[5] P. M. Cohn, Some remarks on the invariant basis property, Topology 5 (1966), 215-228, Zbl 0147.28802 MR 197511.

[6] Guillermo Cortiñas, Algebraic v. topological K-theory: a friendly match, Topics in algebraic and topological $K$-theory, Lecture Notes in Math., vol. 2008, Springer, Berlin, 2011, pp. 103-165, Zbl 1216.19002 MR 2762555.

[7] Guillermo Cortiñas, Jorge Alberto Guccione, and Juan José Guccione, Decomposition of Hochschild and cyclic homology of commutative differential graded algebras, J.of Pure and Appl. Alg. 83 (1992), 219-235, Zbl 0771.13006 MR 1194838.

[8] Guillermo Cortiñas and Andreas Thom, Bivariant algebraic K-theory, J. Reine Angew. Math. 610 (2007), 71-123, Zbl 1152.19002 MR 2359851.

[9] Guillermo Cortiñas and Andreas Thom, Comparison between algebraic and topological K-theory of locally convex algebras, Adv. Math. 218 (2008), no. 1, 266-307, Zbl 1142.19002 MR 2409415.

[10] Guillermo Cortiñas and Andreas Thom, Algebraic geometry of topological spaces I, Acta Math. 209 (2012), no. 1, 83-131, Zbl 1266.19003 MR 2979510, DOI 10.1007/s11511012-0082-6.

[11] G. Cortiñas and C. Weibel, Relative Chern characters for nilpotent ideals, Algebraic topology, Abel Symp., vol. 4, Springer, Berlin, 2009, pp. 61-82, Zbl 05645847 MR 2597735.

[12] Joachim Cuntz, Ralf Meyer, and Jonathan M. Rosenberg, Topological and bivariant $K$ theory, Oberwolfach Seminars, vol. 36, Birkhäuser Verlag, Basel, 2007, Zbl 1139.19001 MR 2340673.

[13] Ken Dykema, Tadeusz Figiel, Gary Weiss, and Mariusz Wodzicki, Commutator structure of operator ideals, Adv. Math. 185 (2004), no. 1, 1-79, Zbl 1103.47054 MR 2058779.

[14] Ruy Exel, Inverse semigroups and combinatorial $C^{*}$-algebras, Bull. Braz. Math. Soc. (N.S.) 39 (2008), no. 2, 191-313, Zbl 1173.46035 MR 2419901.

[15] D. J. H. Garling, On ideals of operators in Hilbert space, Proc. London Math. Soc. (3) 17 (1967), 115-138, Zbl 0149.34202 MR 208398. 
[16] M. Khalkhali and B. Rangipour, On the generalized cyclic Eilenberg-Zilber theorem, Canad. Math. Bull. 47 (2004), no. 1, 38-48, Zbl 1048.19004 MR 2032267.

[17] Jean-Louis Loday, Cyclic homology, 2nd ed., Grundlehren der Mathematischen Wissenschaften, vol. 301, Springer-Verlag, Berlin, 1998, Zbl 0885.18007 MR 1600246.

[18] Wolfgang Lück, Hilbert modules and modules over finite von Neumann algebras and applications to $L^{2}$-invariants, Math. Ann 309 (1997), 247-285, Zbl 0886.57028 MR 1474192.

[19] Daniel Quillen, Cyclic cohomology and algebra extensions, K-Theory 3 (1989), no. 3, 205-246, Zbl 0696.16021 MR 1040400.

[20] Andrei A. Suslin and Mariusz Wodzicki, Excision in algebraic K-theory, Ann. of Math. (2) 136 (1992), no. 1, 51-122, Zbl 0756.18008 MR 1173926.

[21] Micheline Vigué-Poirrier, Décompositions de l'homologie cyclique des algèbres diférentielles graduées commutatives, K-Theory 4 (1991), no. 5, 399-410, Zbl 0731.19004 MR 1116926.

[22] Charles A. Weibel, An introduction to homological algebra, Cambridge Studies in Advanced Mathematics, vol. 38, Cambridge University Press, Cambridge, 1994, Zbl 1269324 MR 1269324.

[23] Mariusz Wodzicki, Excision in cyclic homology and in rational algebraic K-theory, Ann. of Math. (2) 129 (1989), no. 3, 591-639, Zbl 0689.16013 MR 997314.

[24] Mariusz Wodzicki, Algebraic K-theory and functional analysis, First European Congress of Mathematics, Vol. II (Paris, 1992), Progr. Math., vol. 120, Birkhäuser, Basel, 1994, pp. 485-496, Zbl 0842.46047 MR 1341858.

Received 12 April, 2013

G. Cortiñas, Dep. Matemática-IMAS, FCEyN-UBA, Ciudad Universitaria Pab 1, 1428

Buenos Aires, Argentina

E-mail: gcorti@dm.uba.ar 